\title{
Balancing Objective Optimization and Constraint Satisfaction in Constrained Evolutionary Multi-Objective Optimization
}

\author{
Ye Tian, Yajie Zhang, Yansen Su, Xingyi Zhang, Senior Member, IEEE, \\ Kay Chen Tan, Fellow, IEEE, and Yaochu Jin, Fellow, IEEE
}

\begin{abstract}
Both objective optimization and constraint satisfaction are crucial for solving constrained multi-objective optimization problems, but existing evolutionary algorithms encounter difficulties in striking a good balance between them when tackling complex feasible regions. To address this issue, this paper proposes a two-stage evolutionary algorithm, which adjusts the fitness evaluation strategies during the evolutionary process to adaptively balance objective optimization and constraint satisfaction. The proposed algorithm can switch between the two stages according to the status of the current population, enabling the population to cross the infeasible region and reach the feasible regions in one stage, and to spread along the feasible boundaries in the other stage. Experimental studies on four benchmark suites and three real-world applications demonstrate the superiority of the proposed algorithm over the state-of-theart algorithms, especially on problems with complex feasible regions.
\end{abstract}

Index Terms-Constrained multi-objective optimization problems, evolutionary algorithm, objective optimization, constraint satisfaction

Manuscript received -. This work was supported in part by National Key R\&D Program of China under Grant 2018AAA0100100, in part by the National Natural Science Foundation of China under Grant 61672033, Grant 61822301, Grant 61876123, Grant 61906001, Grant 61590922, and Grant U1804262, in part by the Hong Kong Scholars Program under Grant XJ2019035, in part by the Anhui Provincial Natural Science Foundation under Grant 1808085J06 and Grant 1908085QF271, in part by the State Key Laboratory of Synthetical Automation for Process Industries under Grant PAL-N201805, in part by the Research Grants Council of the Hong Kong Special Administrative Region, China under Grant CityU11202418 and Grant CityU11209219, and in part by a Royal Society International Exchanges Program under Grant IEC $\backslash$ NSFC $\backslash 170279$. (Corresponding authors: Yansen Su and Xingyi Zhang.)

Y. Tian is with the Key Laboratory of Intelligent Computing and Signal Processing of Ministry of Education, Institutes of Physical Science and Information Technology, Anhui University, Hefei 230601, China (email: field910921@gmail.com).

Y. Zhang, Y. Su, and X. Zhang are with the Key Laboratory of Intelligent Computing and Signal Processing of Ministry of Education, School of Computer Science and Technology, Anhui University, Hefei 230601, China (email: yjzhang17719490727@163.com; suyansen1985@163.com; xyzhanghust@gmail.com).

K. C. Tan is with the Department of Computer Science, City University of Hong Kong, Kowloon Tong, Hong Kong SAR (email: kaytan@cityu.edu.hk).

Y. Jin is with the Department of Computer Science, University of Surrey, Guildford, Surrey, GU2 7XH, U.K., and also with the Department of Computer Science and Engineering, Southern University of Science and Technology, Shenzhen 518055, China (email: yaochu.jin@surrey.ac.uk).

\section{INTRODUCTION}

$\mathbf{T}$ HERE are many real-world optimization problems containing multiple objectives and constraints. For instance, the time-varying ratio error estimation problem aims to minimize the total ratio error and its variation, and the solutions should meet the constraints of topology, series, and phase [1]. The robot gripper optimization problem aims to minimize the fluctuation of gripping force and the force transmission ratio, and the solutions are restricted by six constraints related to geometry and force considerations [2]. These problems are collectively known as constrained multi-objective optimization problems (CMOPs), which can be mathematically defined as

$$
\begin{array}{ll}
\text { Minimize } & \mathbf{F}(\mathbf{x})=\left(f_{1}(\mathbf{x}), \cdots, f_{m}(\mathbf{x})\right) \\
\text { subject to } & \mathbf{x} \in \Omega \\
& g_{i}(\mathbf{x}) \leq 0, \quad i=1, \cdots, p \\
& h_{j}(\mathbf{x})=0, \quad j=1, \cdots, q
\end{array}
$$

where $\mathbf{x}=\left(x_{1}, \cdots, x_{d}\right) \in \Omega$ is the solution consisting of $d$ decision variables, $\Omega \subseteq \mathbb{R}^{d}$ is the decision space, $\mathbf{F}: \Omega \rightarrow \mathbb{R}^{m}$ consists of $m$ objectives, and $g_{i}(\mathbf{x})$ and $h_{j}(\mathbf{x})$ are $p$ inequality and $q$ equality constraints, respectively.

Over the last two decades, evolutionary algorithms have been widely employed to solve multi-objective optimization problems, which are able to strike a balance between the conflicting objectives and gain a population with good convergence and diversity. For example, the Pareto dominance based evolutionary algorithms improve the population convergence by non-dominated sorting [3] and maintain the population diversity by various selection strategies [4]. The decomposition based evolutionary algorithms predefine a set of uniformly distributed weight vectors to ensure the population diversity [5], and drive the population to converge along the weight vectors by optimizing aggregation functions [6]. The indicator based evolutionary algorithms select solutions according to their contributions to performance indicators like HV [7] and IGD-NS [8], where performance indicators can evaluate the convergence and diversity of a population simultaneously. In order to solve CMOPs, some constraint handling techniques should be additionally considered for balancing the objectives and constraints, such as the constraint dominance principle 

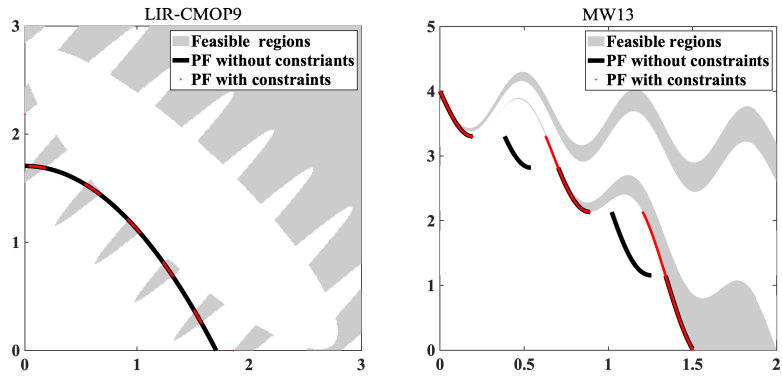

Fig. 1. Feasible regions of LIR-CMOP9 and MW13. The objectives should be given an equal or higher priority than the constraints for solving LIR-CMOP9 and given a lower priority than the constraints for solving MW13.

of NSGA-II-CDP [9], the two-archive strategy of C-TAEA [10], and the push-and-pull search process of PPS [11].

In spite of the promising performance of existing constrained multi-objective evolutionary algorithms (CMOEAs) on simple CMOPs, they are incapable of balancing objective optimization and constraint satisfaction on complex CMOPs [12]-[14], whose feasible regions may be disconnected (e.g., LIR-CMOP9 [12]) or disjoint from the unconstrained Pareto front (e.g., MW13 [13]) as shown in Fig. 1. In fact, for the CMOPs with disconnected feasible regions, the objectives can be given an equal or higher priority than the constraints, so that the solutions can cross the infeasible regions and reach the feasible regions. While for the CMOPs whose feasible regions are disjoint from the unconstrained Pareto front, the objective can be given a lower priority than the constraints, so that the solutions can stay in the feasible region for better diversity. As a consequence, it is unreasonable to use a single constraint handling technique to solve different CMOPs; by contrast, the priority of objectives and constraints should be adaptively adjusted to adapt to various feasible regions.

Following this idea, this paper proposes a two-stage CMOEA to adaptively balance objective optimization and constraint satisfaction during the evolutionary process. To be more specific, the main contributions of this work can be summarized as follows:

1) A two-stage CMOEA is proposed for solving CMOPs with complex feasible regions. The proposed algorithm divides the evolutionary process into two stages, and uses different fitness evaluation strategies in the two stages to adjust the priority of objectives and constraints. The Stage A indicates that the solutions are mostly infeasible, and the objectives are given the same priority to constraints for reaching all the feasible regions. By contrast, the Stage B indicates that the solutions are mostly feasible, and the objectives are given a lower priority than the constraints for diversifying the solutions along the feasible boundaries. The proposed algorithm automatically determines the current stage according to the status of the population at each generation, which is versatile for tackling various feasible regions.
2) Several case studies on benchmark CMOPs are performed to reveal the importance of adaptively balancing objective optimization and constraint satisfaction in solving CMOPs with different feasible regions. Moreover, a series of experiments on challenging benchmark suites and real-world applications are conducted to verify the superiority of the proposed CMOEA over the state-of-the-art, especially on CMOPs with complex feasible regions.

The rest of this paper is organized as follows. In Section II, we briefly review the state-of-the-art CMOEAs according to the strategies for balancing objectives and constraints, and then we elicit the motivation of this work. The proposed algorithm is elaborated in Section III, followed by a description of the experimental studies in Section IV. Finally, the conclusions and future work are presented in Section V.

\section{RELATED WORK AND MOTIVATION}

\section{A. Existing CMOEAs}

In general, existing CMOEAs can be grouped into the following four categories, where the priorities of objectives and constraints are different in these categories.

The first category considers objectives and constraints equally. In [15], the objective vector of each solution is extended with the violation of each constraint, then the non-dominated sorting is performed on the extended objective vectors. Inspired by [16], the algorithm in [17] performs non-dominated sorting on objectives and constraints separately, then blends the two non-dominated ranks. In [18], the objective values of each solution are modified by considering its constraint violation. In [19], a constrained non-dominated rank is defined by integrating the original non-dominated rank and a constraint rank. In [20], a tri-goal evolutionary framework is proposed to consider convergence, diversity, and feasibility.

The second category makes constraints always prior to objectives. The algorithm in [21] directly discards infeasible solutions, which may lose selection pressure when most solutions are infeasible. NSGA-II [9] embeds feasibility dominance principle into Pareto dominance for solving CMOPs, which is known as NSGA-II-CDP. A similar strategy is extended to MOEA/D, which prefers constraint satisfaction than aggregation function in updating the solution for each weight vector [22]. The constraint handling strategies in NSGA-II and MOEA/D can also be easily applied to most Pareto dominance based MOEAs and decomposition based MOEAs [23], respectively, but such an excessive use of feasibility may make the population trap into a small feasible region. To relax the definition of feasibility, the algorithm in [24] regards solutions with small constraint violations as feasible ones, the algorithm in [12] regards solutions with constraint violations smaller than a predefined parameter $\varepsilon$ as feasible ones, the algorithm in [25] makes no difference when the constraint violations of two solutions are similar, the algorithm in [26] treats two infeasible 
solutions as non-dominated if the angle between them is large, and the algorithm in [27] preserves the infeasible solutions in isolated regions. In addition to the use of constraint violation, the number of violated constraints [28] and the dominance relation based on constraints [29], [30] have also been considered.

The third category balances objectives and constraints dynamically by the use of different strategies in different populations. The DyHF framework [31] divides the population into several subpopulations according to the Euclidean distances between solutions, and then performs global search or local search dynamically. In [32], the algorithm also divides the population into several subpopulations and considers a single constraint in each subpopulation. The DPDE framework [33] divides the population into two subpopulations, hoping one population to evolve from infeasible regions to feasible regions and the other population to be optimized in the feasible regions. Similarly, the algorithm in [34] evolves one population by optimizing only the objectives, and evolves another population by optimizing only the constraints. In C-TAEA [10], a convergence-oriented archive is evolved by optimizing both the objectives and constraints, and a diversity-oriented archive is evolved by optimizing only the objectives.

The fourth category balances objectives and constraints dynamically through different strategies at different stages of the search process. ToPDE [35] tries to find out as many feasible solutions as possible in the first stage, and optimizes those feasible solutions in the second stage. In [36], [37], two novel frameworks are proposed for constrained optimization, which convert the original constrained problem to an equivalent dynamic CMOP and solve it via dynamic multi-objective evolutionary algorithms, where the constraint boundary is gradually reduced to the original constraint boundary in the dynamic CMOP. The PPS framework [11] divides the search process into a push stage and a pull stage, where the population is evolved without considering constraints in the push stage and with considering all the objectives and constraints in the pull stage. The ToP framework [14] also suggests a two-stage search process, where all the constraints and a single objective are considered in the first stage, and all the constraints and objectives are considered in the second stage.

\section{B. Motivation of This Work}

It can be found that the balance between objective optimization and constraint satisfaction is a key issue in solving CMOPs. However, most existing CMOEAs encounter difficulties in striking a good balance between objectives and constraints when solving CMOPs with complex feasible regions [13], [14]. To better illustrate the limitations of existing CMOEAs, Fig. 2 plots the populations obtained by NSGA-II-CDP, C-TAEA, and PPS on LIR-CMOP9 and MW13, where the parameter settings are presented in Section IV-B. As mentioned
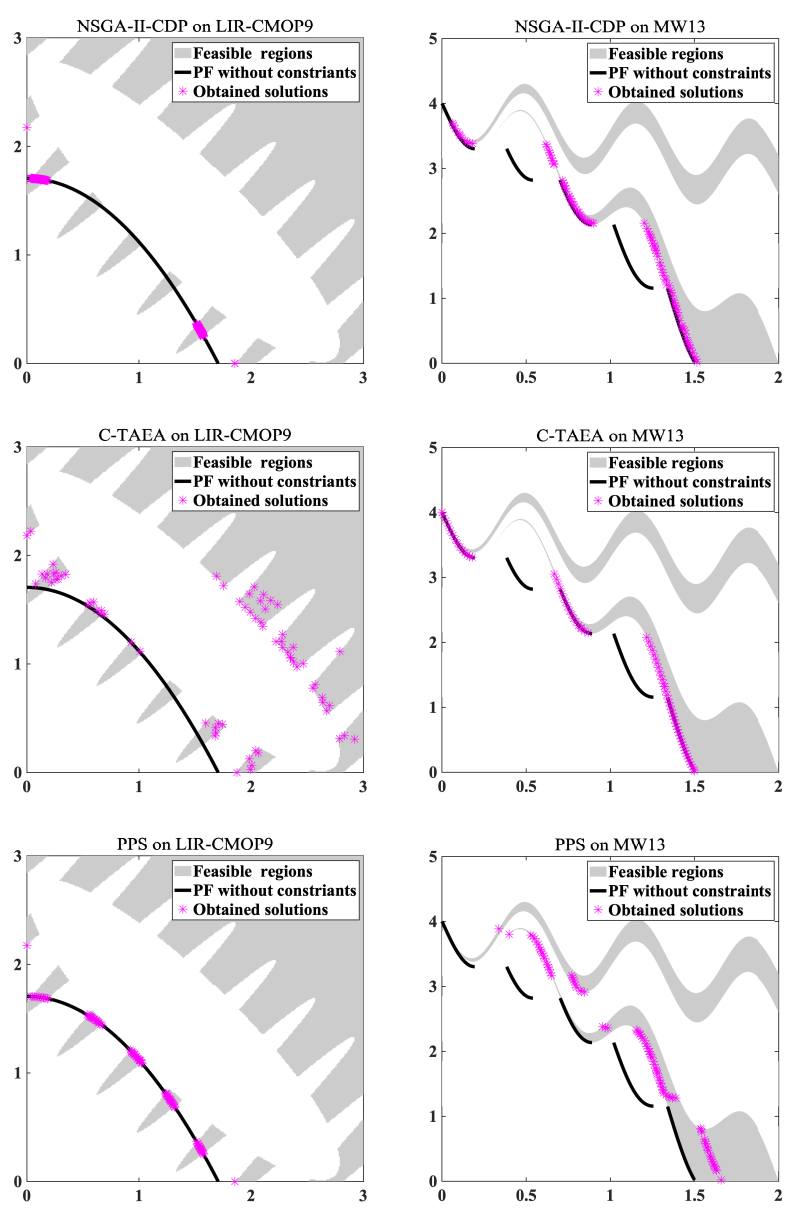

Fig. 2. Populations obtained by NSGA-II-CDP, C-TAEA, and PPS on LIR-CMOP9 and MW13. NSGA-II-CDP and C-TAEA perform well on only MW13, while PPS performs well on only LIR-CMOP9.

before, the objectives should be given an equal or higher priority than the constraints for solving LIR-CMOP9, while the objectives should be given a lower priority than the constraints for solving MW13.

For LIR-CMOP9 whose feasible regions are disconnected and separated by a large infeasible region, the population of NSGA-II-CDP converges to a few feasible regions since it always prefers feasible solutions than infeasible solutions (i.e., the priority of objectives is lower than constraints), which disables the population from crossing the infeasible region and reaching all the feasible regions. Similarly, many solutions obtained by C-TAEA cannot cross the infeasible region since the main population (i.e., convergence-oriented archive) also prefers feasible solutions than infeasible solutions. Besides, the solutions obtained by PPS can reach all the feasible regions and converge well, since it considers only the objectives in the push stage and considers both the objectives and constraints in the pull stage (i.e., the objectives always have an equal or higher priority than the constraints). On the other hand, for MW13 whose feasible regions are disjoint from the unconstrained Pareto front, the populations of NSGA-II-CDP and CTAEA have good convergence and diversity, since they give a lower priority to the objectives that enable the 


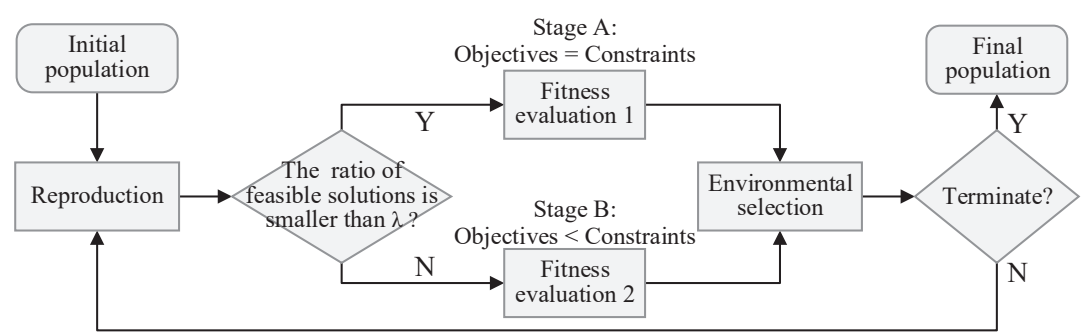

Fig. 3. Procedure of the proposed CMOEA-MS.
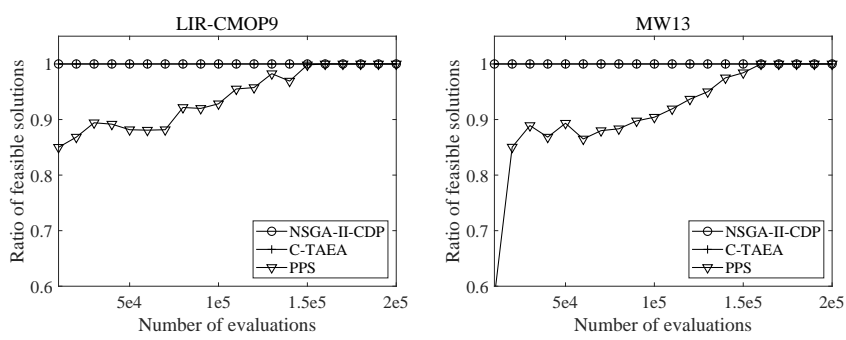

Fig. 4. Ratio of feasible solutions in the populations obtained by NSGA-II-CDP, C-TAEA, and PPS on LIR-CMOP9 and MW13. The three CMOEAs cannot perform well on both LIR-CMOP9 and MW13 since the priority of objectives and constraints (reflected by the ratio of feasible solutions) remains unchanged on the two CMOPs.

solutions to spread along the feasible boundary. By contrast, the population of PPS has worse convergence than those of NSGA-II-CDP and C-TAEA, which is mainly due to the high priority of the objectives.

As a consequence, none of the three CMOEAs can balance the objectives and constraints on both LIRCMOP9 and MW13, since the objectives are always given a lower priority than the constraints in NSGA-II-CDP and C-TAEA, and the objectives are always given an equal or higher priority than the constraints in PPS. For further observation, Fig. 4 depicts the ratio of feasible solutions in the populations at each generation of the three CMOEAs averaged over 30 runs, where a higher ratio of feasible solutions indicates a lower priority of the objectives. It can be found that the ratio of feasible solutions in the populations of NSGA-II-CDP and C-TAEA is always 1, which is beneficial for solving MW13 but ineffective for solving LIR-CMOP9. By contrast, the ratio of feasible solutions in the population of PPS is smaller than 1 since it gives the objectives an equal priority to the constraints, which is improper for solving MW13. Note that the ratio of feasible solutions of PPS reaches 1 at last since PPS gives the objectives a lower priority than the constraints at the last several generations, but these generations are not enough to spread the population along the feasible boundary of MW13.

To address the limitations of existing CMOEAs, the proposed CMOEA automatically adjusts the priority of objectives and constraints at different stages. By giving the objectives an equal priority to the constraints in one stage and a lower priority than the constraints in the other stage, the proposed CMOEA is expected to tackle CMOPs with different feasible regions. In the next section, the procedure of the proposed CMOEA is described.

\section{The Proposed Algorithm}

\section{A. Procedure of CMOEA-MS}

Fig. 3 illustrates the procedure of the proposed CMOEA with a two-stage framework (CMOEA-MS). The proposed algorithm first generates an initial population randomly, then repeats reproduction, fitness evaluation, and environmental selection until the termination condition is satisfied. The novelty of CMOEA-MS mainly lies in the two-stage framework, which automatically determines the current stage at each generation and uses different fitness evaluation strategies in the two stages for balancing the objectives and constraints. More specifically, if the ratio of feasible solutions in the combined population is smaller than $\lambda$, CMOEA-MS will use the fitness evaluation strategy of Stage A to give the objectives the same priority to the constraints, hence some infeasible solutions can be maintained to help the population cross infeasible regions. Otherwise, CMOEAMS will use the fitness evaluation strategy of Stage B to give the objectives a lower priority than the constraints, hence more feasible solutions can be found to help the population spread along the feasible boundaries.

As presented in Algorithm 1, CMOEA-MS starts with the random initialization of a population with size $n$. In the reproduction procedure, $n$ parents are selected from the current population $P$ by binary tournament selection based on the fitness of solutions, then $n$ offspring solutions are generated based on the parents and combined with the current population. Afterwards, the algorithm will enter either Stage A or Stage B. In the environmental selection procedure, $n$ solutions with better fitness values are selected and survive to the next generation; as suggested in [38], if the fitness values of more than $n$ solutions in $P$ are smaller than 1 (i.e., these solutions are non-dominated), $n$ of them are selected by the truncation method based on the Euclidean distances between solutions. In other words, the solutions with fitness values larger than 1 are compared according to their fitness values, and the solutions with fitness values smaller than 1 are compared according to the Euclidean distances between them.

As a consequence, the fitness of solutions plays a crucial role in the proposed algorithm, where the fitness evaluation strategies in the two stages are also the core 


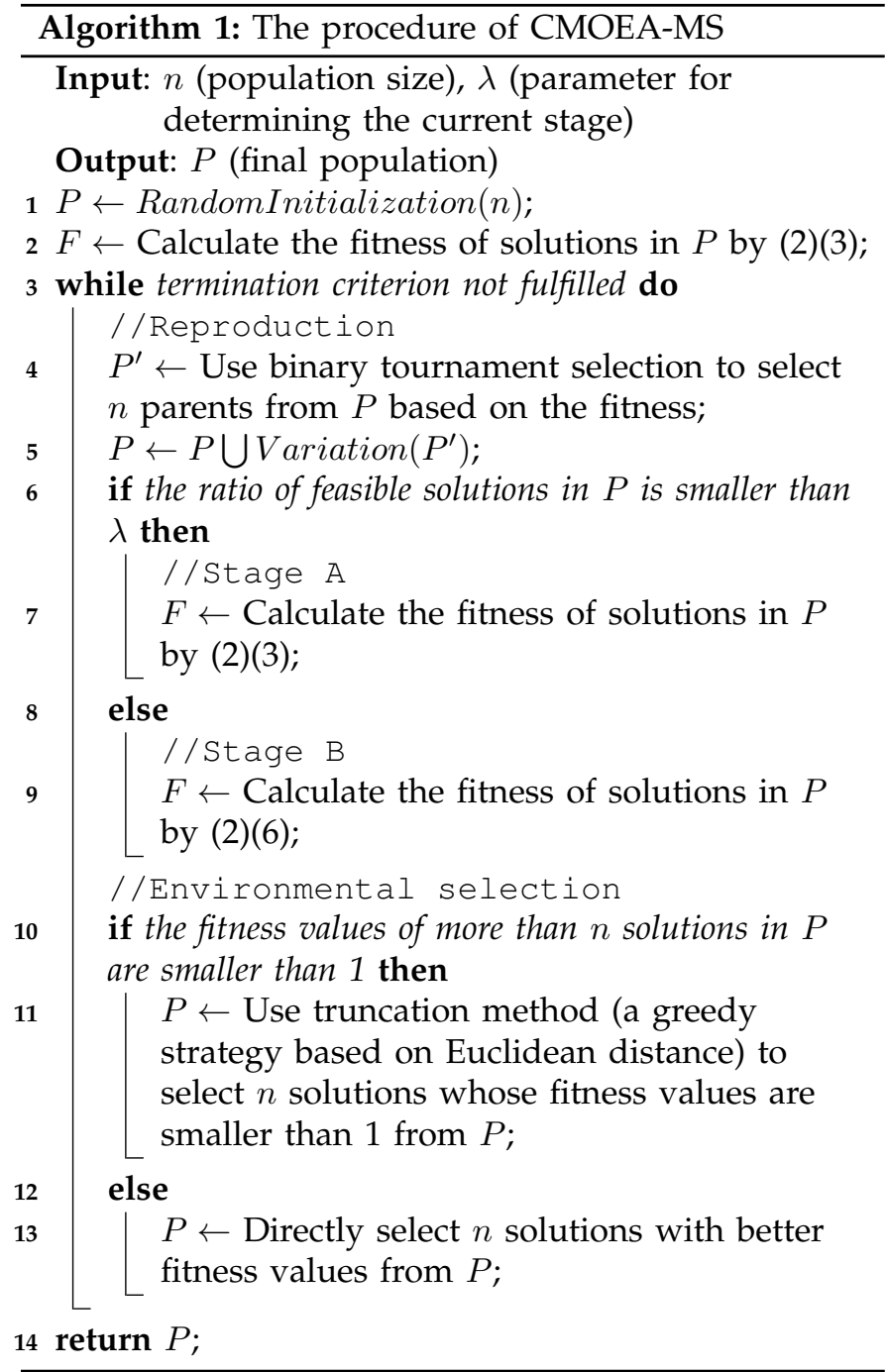

contributions of this work. In the next subsection, the details of the proposed fitness evaluation strategies are elaborated.

\section{B. Fitness Evaluation Strategies in CMOEA-MS}

The fitness evaluation strategies in CMOEA-MS hold a similar idea to the one in SPEA2 [38]. First of all, the solution set $R_{\mathrm{x}}$ storing all the solutions dominated by $\mathrm{x}$ and the solution set $S_{\mathrm{x}}$ storing all the solutions dominating $\mathbf{x}$ are determined, and the $\lfloor\sqrt{2 n}\rfloor$-th nearest neighbor $\mathbf{x}^{\prime}$ of $\mathbf{x}$ is found. Then, the fitness of solution $\mathrm{x}$ can be calculated by

$$
f i t(\mathbf{x})=\sum_{\mathbf{y} \in S_{\mathbf{x}}}\left|R_{\mathbf{y}}\right|+\frac{1}{\operatorname{dist}\left(\mathbf{x}, \mathbf{x}^{\prime}\right)+2},
$$

where the first component of $f i t(\mathbf{x})$ counts the total number of solutions dominated by the solutions dominating $\mathrm{x}$, and the second component calculates the inverse of the Euclidean distance from $\mathbf{x}$ to its $\lfloor\sqrt{2 n}\rfloor$ th nearest neighbor. Obviously, a smaller fitness value represents a better quality of the solution, and $f i t(\mathbf{x})<1$ indicates that the solution is not dominated by any others.

The difference between the proposed fitness evaluation strategies and the one in SPEA2 lies in the dominance relation for determining $R_{\mathbf{x}}$ and $S_{\mathbf{x}}$. To be more specific, SPEA2 uses the conventional Pareto dominance [39] to determine the dominance relation between each two solutions, which considers all the objectives of solutions. By contrast, the Stage A of CMOEA-MS determines the dominance relation by considering the following two new objectives:

$$
\begin{array}{ll}
\text { Minimize } & \mathbf{F}^{\prime}(\mathbf{x})=(S D E(\mathbf{x}), C V(\mathbf{x})) \\
\text { subject to } & \mathbf{x} \in \Omega
\end{array}
$$

where $S D E(\mathbf{x})$ is the minimum shift-based density estimation based distance between $\mathrm{x}$ and other solutions in the population $P$ [40]:

$$
S D E(\mathbf{x})=\min _{\mathbf{y} \in P \backslash\{\mathbf{x}\}} \sqrt{\sum_{i=1}^{m}\left(\max \left\{0, f_{i}(\mathbf{y})-f_{i}(\mathbf{x})\right\}\right)^{2}},
$$

and $C V(\mathbf{x})$ is the total constraint violation of $\mathbf{x}$ [9]:

$$
C V(\mathbf{x})=\sum_{i=1}^{p} \max \left\{g_{i}(\mathbf{x}), 0\right\}+\sum_{j=1}^{q}\left|h_{j}(\mathbf{x})\right|,
$$

where $f_{i}(\mathbf{x}), g_{i}(\mathbf{x})$, and $h_{i}(\mathbf{x})$ denote the objective values, inequality constraints, and equality constraints of the solution, respectively. The first objective $S D E(\mathbf{x})$ can evaluate the quality of a solution in terms of both convergence and diversity, and the second objective $C V(\mathbf{x})$ evaluates the quality of a solution in terms of constraint satisfaction. Therefore, the Stage A of CMOEA-MS gives the objectives an equal priority to the constraints. Besides, in contrast to some existing CMOEAs consider the sum of all the objectives as the first objective [14], the use of shift-based density estimation based distance can additionally consider the population diversity to enable the solutions to reach the isolated feasible regions. The experiments in Section IV-E will demonstrate the superiority of shift-based density estimation based distance over the sum of all the objectives.

On the other hand, the Stage B of CMOEA-MS aims to give the objectives a lower priority than the constraints. To this end, the constraint dominance principle [9] is adopted in the Pareto dominance to determine the dominance relation between each two solutions. More formally, a solution $\mathrm{x}$ dominates another solution $\mathrm{y}$ if the following conditions hold:

$$
\begin{aligned}
& \left\{\begin{array}{l}
C V(\mathbf{x})=0 \\
C V(\mathbf{y})=0 \\
f_{i}(\mathbf{x}) \leq f_{j}(\mathbf{y}), \forall i \in\{1, \ldots, m\} \\
f_{i}(\mathbf{x})<f_{j}(\mathbf{y}), \quad \exists i \in\{1, \ldots, m\}
\end{array}\right. \\
& \quad \text { or } \\
& C V(\mathbf{x})<C V(\mathbf{y})
\end{aligned}
$$



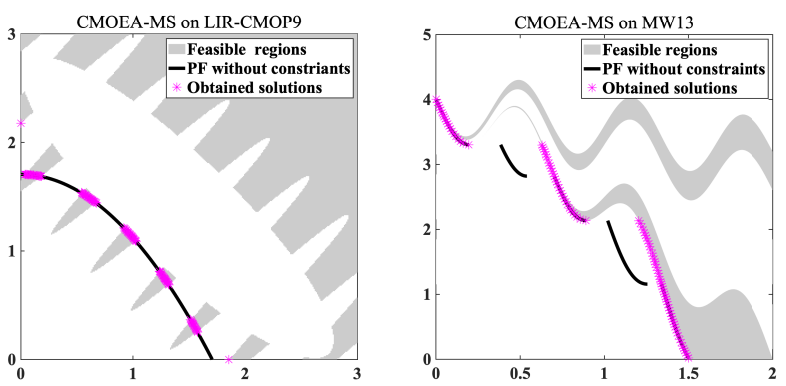

Fig. 5. Populations obtained by the proposed CMOEA-MS on LIRCMOP9 and MW13. CMOEA-MS performs well on both the two CMOPs.

As a result, the Stage B always prefers the solution with lower constraint violation; while if both the two solutions are feasible, they are compared based on the conventional Pareto dominance.

\section{Analysis of CMOEA-MS}

To investigate the performance of CMOEA-MS and verify whether it can balance objective optimization and constraint satisfaction on different CMOPs, it is tested on LIR-CMOP9 and MW13 with $\lambda=0.5$. As can be seen from Fig. 5, the population can not only reach all the feasible regions of LIR-CMOP9 but also uniformly spread along the feasible boundary of MW13. In short, CMOEA-MS can better balance objective optimization and constraint satisfaction than NSGA-II-CDP, C-TAEA, and PPS on the two CMOPs.

The superiority of CMOEA-MS is attributed to the different priorities of objectives and constraints considered in the two stages. When solving LIR-CMOP9 with many small and isolated feasible regions, CMOEA-MS enters Stage A since most offspring solutions are likely to locate in the infeasible region, hence the objectives are given an equal priority to the constraints. On the contrary, when solving MW13 whose feasible regions are continuous but disjoint from the unconstrained Pareto front, CMOEAMS enters Stage B since most offspring solutions are likely to locate in the feasible region, hence the objectives are given a lower priority than the constraints. For further demonstration, Fig. 6 plots the ratio of feasible solutions in the populations at each generation of CMOEA-MS, averaged over 30 runs. It can be found that the ratios of feasible solutions on the two CMOPs are quite different, which means that CMOEA-MS uses different priorities to the objectives and constraints. Specifically, the low ratio on LIR-CMOP9 demonstrates that CMOEA-MS is always in Stage A that gives the objectives an equal priority to the constraints, and the high ratio on MW13 demonstrates that CMOEA-MS is mostly in Stage B that gives the objectives a lower priority than the constraints. By contrast, the ratio of feasible solutions in the populations of NSGA-II-CDP, CTAEA, and PPS remains unchanged on LIR-CMOP9 and MW13, which is unable to balance objective optimization and constraint satisfaction on both the two CMOPs.
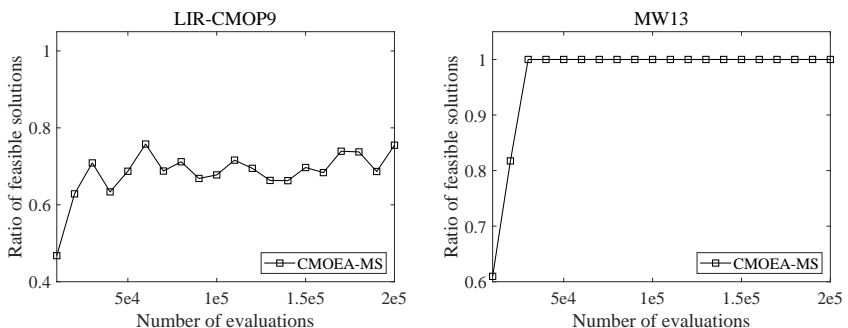

Fig. 6. Ratio of feasible solutions in the populations obtained by the proposed CMOEA-MS on LIR-CMOP9 and MW13. CMOEA-MS performs well on both LIR-CMOP9 and MW13 since the priority of objectives and constraints (reflected by the ratio of feasible solutions) adapts to the two CMOPs.
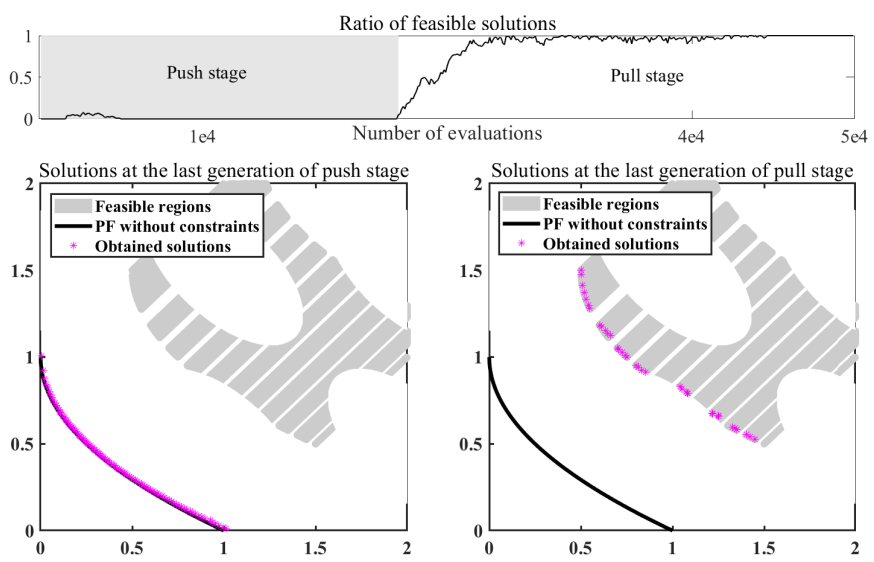

(a) PPS on DAS-CMOP5
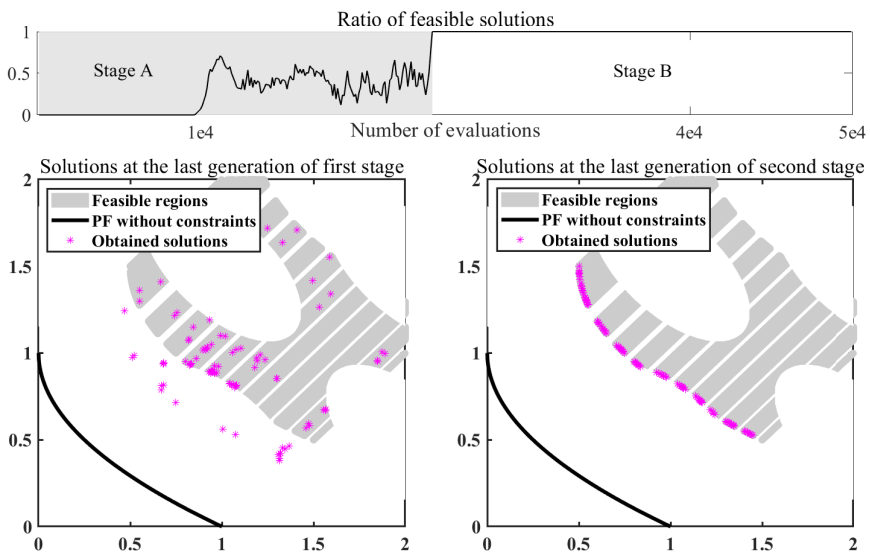

(b) CMOEA-MS on DAS-CMOP5

Fig. 7. Populations and ratio of feasible solutions of PPS and the proposed CMOEA-MS on DAS-CMOP5. CMOEA-MS obtains a better population than PPS at the last generation.

Fig. 7 illustrates the search behaviors of PPS and the proposed CMOEA-MS $(\lambda=0.5)$ in a more complex scenario, where the feasible regions of the problem DASCMOP5 [41] have the difficulties of both LIR-CMOP9 and MW13. That is, the population should be able to cross the infeasible region, reach the isolated feasible regions, and spread along the feasible boundary at last. As can be seen from the figure, CMOEA-MS obtains a better population than PPS at last, which is mainly due to two reasons: Firstly, the population converges to the unconstrained Pareto front in the push stage of PPS since 
the constraints are not considered at all, hence much effort should be made to pull the population back to the feasible regions; by contrast, the population remains in the feasible regions in Stage A of CMOEA-MS since both the objectives and constraints are considered. Secondly, the ratio of feasible solutions in the pull stage of PPS is not always 1 , which means that some infeasible solutions are preferred and hinder the population in spreading along the feasible boundary; by contrast, the ratio of feasible solutions in Stage B of CMOEA-MS is always 1 , which means that no infeasible solution is preferred and the population can easily spread along the feasible boundary. As a result, CMOEA-MS is more effective than PPS in tackling the feasible regions that are disconnected and far from the unconstrained Pareto front.

To give a deeper understanding of the advantage of CMOEA-MS, the search behaviors of NSGA-II-CDP, CTAEA, PPS, and CMOEA-MS $(\lambda=0.8)$ are investigated on the following CMOP:

$$
\begin{aligned}
\text { Minimize } & \mathbf{F}(\mathbf{x})=\left(f_{1}(\mathbf{x}), f_{2}(\mathbf{x})\right) \\
\text { where } & f_{1}(\mathbf{x})=(1+g(\mathbf{x})) \cos \left(x_{1}\right) \\
& f_{2}(\mathbf{x})=(1+g(\mathbf{x})) \sin \left(x_{1}\right) \\
& g(\mathbf{x})=10 \sum_{i=2}^{50}\left(x_{i}-0.5\right)^{2} \\
\text { subject to } & \mathbf{x} \in[0,1]^{50} \\
& \cos (0.1 \pi g(\mathbf{x})) \leq 0
\end{aligned}
$$

Obviously, the unconstrained Pareto front is $f_{1}^{2}+f_{2}^{2}=1$ while the feasible boundary is $f_{1}^{2}+f_{2}^{2}=36$, and the feasible regions and infeasible regions are alternatively arranged with the increase of $g(\mathbf{x})$ as shown in Fig. 8 . Fig. 8 also shows the worst value of $g(\mathbf{x})$ among all the solutions in the populations at each generation of the four CMOEAs, averaged over 30 runs. For NSGAII-CDP, the population is hindered by the infeasible regions and cannot reach the feasible boundary. For CTAEA and CMOEA-MS, both their populations can reach the feasible boundary, and the population of CMOEAMS converges much faster than the population of CTAEA. For PPS, although the population can reach the feasible boundary at last, much search effort is wasted on approximating the unconstrained Pareto front. In short, CMOEA-MS is more efficient than the other CMOEAs in solving the problem, which is mainly due to the two stages alternately used for reaching the feasible boundary as shown at the bottom of Fig. 8 .

\section{Computational Complexity of CMOEA-MS}

The computational complexity of CMOEA-MS is governed by three procedures at each generation, including reproduction, fitness evaluation, and environmental selection. For reproduction, the time complexities of binary tournament selection and offspring generation are $O(n)$ and $O(d n)$, respectively, where $n$ denotes the population size and $d$ denotes the number of decision variables. For

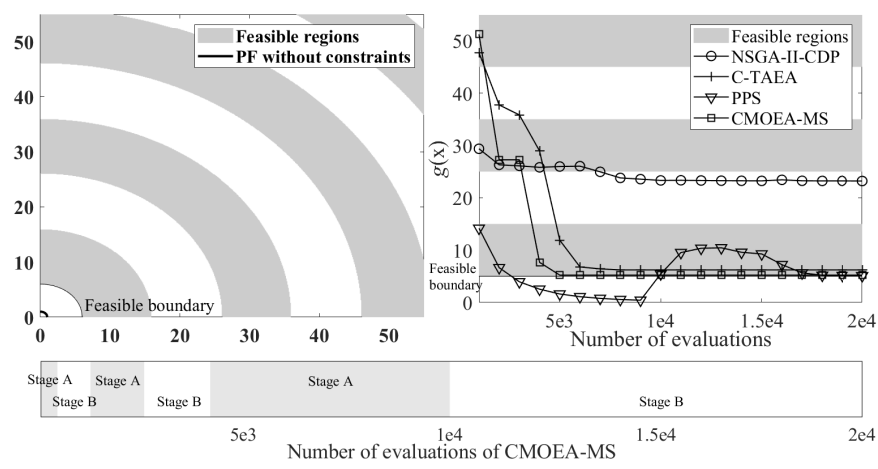

Fig. 8. Feasible regions of the CMOP in (7) and the worst value of $g(\mathbf{x})$ among all the solutions in the populations obtained by NSGAII-CDP, C-TAEA, PPS, and CMOEA-MS. CMOEA-MS is more efficient than the other CMOEAs in reaching the feasible boundary.

fitness evaluation, the time complexities of calculating $S D E$, calculating $C V$, determining the dominance relations, and calculating the fitness of all the solutions are $O\left(m n^{2}\right), O((p+q) n), O\left(m n^{2}\right)$, and $O\left(m n^{2}\right)$, respectively, where $m$ denotes the number of objectives, $p$ denotes the number of inequality constraints, and $q$ denotes the number of equality constraints. For environmental selection, the time complexity of truncation method is $O\left(n^{3}\right)$. To summarize, the total computational complexity of each generation of CMOEA-MS is $O\left(\left(d+m n+p+q+n^{2}\right) n\right)$.

\section{EXPERIMENTAL STUDIES}

A series of experiments are performed in this section to verify the effectiveness of the proposed CMOEAMS in solving CMOPs. Specifically, CMOEA-MS is first compared to five representative CMOEAs on three challenging benchmark suites. Then, the effectiveness of the two-stage framework of CMOEA-MS is verified by ablation study. Lastly, the performance of CMOEA-MS is verified on several CMOPs from real-world applications. All the experiments are conducted on PlatEMO [42].

\section{A. Benchmark Suites}

A number of benchmark CMOPs taken from four recently proposed benchmark suites are adopted as the test problems, which are LIR-CMOP1-LIR-CMOP14 [12], DAS-CMOP1-DAS-CMOP9 [41], MW1-MW14 [13], and DC1-DTLZ1-DC3-DTLZ3 [10]. These CMOPs have various feasible regions, posing stiff challenges for CMOEAs to balance objective optimization and constraint satisfaction. The number of objectives in LIR-CMOP13, LIRCMOP14, DAS-CMOP7, DAS-CMOP8, DAS-CMOP9, MW4, MW8, MW14, and DC1-DTLZ1-DC3-DTLZ3 is 3, the number of objectives in the other CMOPs is 2 , and the number of decision variables in all the CMOPs is set to 10 to ensure that most CMOEAs can converge.

\section{B. Compared Algorithms}

Five popular CMOEAs (i.e., TiGE-2 [20], NSGA-IICDP [9], C-TAEA [10], PPS [11], and ToP [14]) are selected as baselines. TiGE-2 gives the objectives an equal 
TABLE I

Statistical Results of IGD ObTAined by TiGE-2, NSGA-II-CDP, C-TAEA, PPS, ToP, AND the Proposed CMOEA-MS ON the LIR-CMOP BENCHMARK SUITE. BeSt RESUlt IN EACH ROW IS Highlighted.

\begin{tabular}{|c|c|c|c|c|c|c|}
\hline Problem & TiGE-2 & NSGA-II-CDP & C-TAEA & PPS & ToP & CMOEA-MS \\
\hline LIR-CMOP1 & $8.4168 \mathrm{e}-2(2.31 \mathrm{e}-2) \approx$ & $1.8674 \mathrm{e}-1(5.94 \mathrm{e}-2)-$ & $2.4656 \mathrm{e}-1(1.22 \mathrm{e}-1)-$ & $9.4513 \mathrm{e}-3(2.50 \mathrm{e}-3)+$ & $1.6109 \mathrm{e}-1(1.33 \mathrm{e}-1) \approx$ & $9.1974 \mathrm{e}-2(5.02 \mathrm{e}-2)$ \\
\hline LIR-CMOP2 & $1.0482 \mathrm{e}-1(3.20 \mathrm{e}-2) \approx$ & $1.4944 \mathrm{e}-1(5.64 \mathrm{e}-2)-$ & $7.8418 \mathrm{e}-2(1.97 \mathrm{e}-2) \approx$ & $6.4834 \mathrm{e}-3(6.30 \mathrm{e}-4)+$ & $1.5049 \mathrm{e}-1(1.31 \mathrm{e}-1) \approx$ & $9.4703 e-2(4.25 e-2)$ \\
\hline LIR-CMOP3 & $8.7271 \mathrm{e}-2(2.86 \mathrm{e}-2)+$ & $2.4048 \mathrm{e}-1(6.34 \mathrm{e}-2) \approx$ & $2.6444 \mathrm{e}-1(1.78 \mathrm{e}-1) \approx$ & $9.7277 e-3(5.63 e-3)+$ & $3.6618 \mathrm{e}-1(4.55 \mathrm{e}-2)-$ & $2.5920 \mathrm{e}-1(1.37 \mathrm{e}-1)$ \\
\hline LIR-CMOP4 & $9.6294 \mathrm{e}-2(2.38 \mathrm{e}-2)+$ & $2.3352 \mathrm{e}-1(8.92 \mathrm{e}-2) \approx$ & $1.9762 \mathrm{e}-1(1.28 \mathrm{e}-1) \approx$ & $4.9555 \mathrm{e}-3(3.15 \mathrm{e}-3)+$ & $3.3258 \mathrm{e}-1(7.39 \mathrm{e}-2) \approx$ & $2.7395 \mathrm{e}-1(6.20 \mathrm{e}-2)$ \\
\hline LIR-CMOP5 & $2.8706 \mathrm{e}-1(8.80 \mathrm{e}-2)-$ & $7.9890 \mathrm{e}-1(4.53 \mathrm{e}-1)-$ & $9.2948 \mathrm{e}-2(1.55 \mathrm{e}-2)-$ & $6.2936 \mathrm{e}-3(3.99 \mathrm{e}-4)-$ & $2.9253 \mathrm{e}-1(5.17 \mathrm{e}-1)$ & $4.9393 e-3(1.73 e-4)$ \\
\hline LIR-CMOP6 & $5.2491 \mathrm{e}-1(2.13 \mathrm{e}-1)-$ & $5.4141 \mathrm{e}-1(5.21 \mathrm{e}-1)$ & $1.2999 \mathrm{e}-1(1.25 \mathrm{e}-1)-$ & $7.5306 \mathrm{e}-3(8.39 \mathrm{e}-4)-$ & $1.7106 \mathrm{e}-1(3.91 \mathrm{e}-1)$ & $4.9707 \mathrm{e}-3(1.27 \mathrm{e}-4)$ \\
\hline LIR-CMOP7 & $1.5279 \mathrm{e}-1(7.48 \mathrm{e}-2)-$ & $9.8944 \mathrm{e}-3(3.16 \mathrm{e}-3)-$ & $1.8057 \mathrm{e}-2(1.80 \mathrm{e}-3)-$ & $1.0161 \mathrm{e}-2(1.16 \mathrm{e}-3)-$ & $8.6330 \mathrm{e}-3(2.76 \mathrm{e}-4)$ & $7.2675 \mathrm{e}-3(3.34 \mathrm{e}-4)$ \\
\hline LIR-CMOP8 & $2.5226 \mathrm{e}-1(1.48 \mathrm{e}-1)-$ & $7.0191 \mathrm{e}-2(1.20 \mathrm{e}-1)-$ & $1.9867 \mathrm{e}-2(7.30 \mathrm{e}-3)-$ & $1.0574 \mathrm{e}-2(9.05 \mathrm{e}-4)-$ & $8.6143 e-3(3.71 e-4)$ & $7.5092 \mathrm{e}-3(3.56 \mathrm{e}-4)$ \\
\hline LIR-CMOP9 & $7.7370 \mathrm{e}-1(2.47 \mathrm{e}-1)-$ & $5.1760 \mathrm{e}-1(1.39 \mathrm{e}-1)-$ & $6.7924 \mathrm{e}-2(2.68 \mathrm{e}-2)-$ & $3.2808 \mathrm{e}-3(1.32 \mathrm{e}-4)+$ & $2.4650 \mathrm{e}-1(1.79 \mathrm{e}-1) \approx$ & $2.9224 \mathrm{e}-2(9.48 \mathrm{e}-3)$ \\
\hline LIR-CMOP10 & $4.7549 \mathrm{e}-1(2.71 \mathrm{e}-2)-$ & $3.4652 \mathrm{e}-1(9.84 \mathrm{e}-2)-$ & $6.6641 \mathrm{e}-2(5.51 \mathrm{e}-2)-$ & $5.1535 \mathrm{e}-3(1.57 \mathrm{e}-4)-$ & $5.5902 \mathrm{e}-3(2.06 \mathrm{e}-4)-$ & $4.2495 \mathrm{e}-3(1.13 \mathrm{e}-4)$ \\
\hline LIR-CMOP11 & $6.4562 \mathrm{e}-1(3.86 \mathrm{e}-1)-$ & $1.3998 \mathrm{e}-1(1.11 \mathrm{e}-1) \approx$ & $1.2150 \mathrm{e}-1(3.29 \mathrm{e}-2)+$ & $2.3949 \mathrm{e}-3(1.02 \mathrm{e}-4)+$ & $9.6494 \mathrm{e}-2(6.95 \mathrm{e}-2)+$ & $2.3391 \mathrm{e}-1(1.53 \mathrm{e}-1)$ \\
\hline LIR-CMOP12 & $4.4006 \mathrm{e}-1(2.12 \mathrm{e}-1)-$ & $1.5167 \mathrm{e}-1(1.02 \mathrm{e}-1)-$ & $1.3646 \mathrm{e}-2(3.06 \mathrm{e}-3)-$ & $3.0275 \mathrm{e}-3(7.34 \mathrm{e}-5)-$ & $2.8845 \mathrm{e}-3(1.22 \mathrm{e}-4)$ & $2.7937 e-3(5.77 e-5)$ \\
\hline LIR-CMOP13 & $3.5519 \mathrm{e}-1(4.21 \mathrm{e}-2)-$ & $6.7913 \mathrm{e}-2(1.93 \mathrm{e}-3)-$ & $5.4146 \mathrm{e}-2(5.43 \mathrm{e}-4)+$ & $6.7710 \mathrm{e}-2(1.37 \mathrm{e}-3)-$ & $7.7715 \mathrm{e}-2(1.05 \mathrm{e}-3)$ & $6.2303 \mathrm{e}-2(7.74 \mathrm{e}-4)$ \\
\hline LIR-CMOP14 & $3.6556 \mathrm{e}-1(6.10 \mathrm{e}-2)-$ & $7.0478 \mathrm{e}-2(1.61 \mathrm{e}-3)-$ & $5.5815 \mathrm{e}-2(1.04 \mathrm{e}-3)+$ & $6.7115 \mathrm{e}-2(1.18 \mathrm{e}-3)-$ & $7.0685 \mathrm{e}-2(1.16 \mathrm{e}-3)-$ & $5.8666 \mathrm{e}-2(6.46 \mathrm{e}-4)$ \\
\hline$+/-1 \approx$ & $2 / 10 / 2$ & $0 / 11 / 3$ & $3 / 8 / 3$ & $6 / 8 / 0$ & $1 / 9 / 4$ & \\
\hline
\end{tabular}

priority to the constraints, NSGA-II-CDP and C-TAEA give the objectives a lower priority than the constraints, PPS gives the objectives a higher priority than the constraints in the push stage and an equal priority to the constraints in the pull stage, and ToP gives the objectives a lower priority than the constraints in both the first and second stages. According to the taxonomy given in Section II-A, TiGE-2 belongs to the first category, NSGAII-CDP belongs to the second category, C-TAEA belongs to the third category, and PPS and ToP belong to the fourth category. Parameter settings of these CMOEAs are the same as those in their original literatures, which are given in the following; besides, a parameter sensitivity analysis of all the compared CMOEAs is given in Supplementary Materials I.

- The population size of all the CMOEAs is set to 100 for two-objective CMOPs, 300 for three-objective CMOPs, and 330 for five-objective CMOPs.

- The number of function evaluations of all the CMOEAs is set to 200,000 for two-objective CMOPs and 400,000 for three- and five-objective CMOPs.

- TiGE-2, NSGA-II-CDP, C-TAEA, and CMOEA-MS use simulated binary crossover [43] and polynomial mutation [44] to generate offspring solutions, while PPS and ToP use differential evolution [45] and polynomial mutation. The crossover probability is set to 1 , the mutation probability is set to $1 / d(d$ is the number of decision variables), the distribution index of both crossover and mutation is set to 20, and the parameters $C R$ and $F$ in differential evolution are set to 1 and 0.5 , respectively.

- PPS adopts C-MOEA/D [22] as the optimizer, and the other parameter settings are $T_{c}=0.8 \times T_{\max }$, $\alpha=0.95, \tau=0.1, c p=2$, and $l=20$, where $T_{\max }$ denotes the maximum number of generations.

- ToP adopts NSGA-II-CDP as the optimizer, and the first stage ends if the feasibility proportion $P_{f}>1 / 3$ or the difference $\delta<0.2$.

- The parameter $\lambda$ for determining the current stage in CMOEA-MS is set to 0.5 .

\section{Performance Metrics}

Since the constrained Pareto fronts of all the benchmark CMOPs are known, the widely used inverted generational distance (IGD) [46] metric is employed to assess the performance of the compared CMOEAs, where approximately 10,000 uniformly distributed points sampled on each constrained Pareto front are used as the reference points for calculating IGD. While the constrained Pareto fronts of all the real-world CMOPs are unknown, the hypervolume (HV) [47] metric is employed, where the reference point is set to $1.1 \times z^{\text {nad }}\left(z^{\text {nad }}\right.$ denotes the nadir point of the non-dominated solutions obtained by all the CMOEAs) as suggested in [48]. All the CMOEAs are executed on each CMOP for 30 independent runs, and the mean value and standard deviation of the 30 metric values are recorded. Besides, we also take the Wilcoxon rank sum test with a significance level of 0.05 to analyze the results, where ' + ',${ }^{\prime}-$ ', and ' $\approx$ ' indicate that the result of a compared CMOEA is significantly better, significantly worse, and statistically similar to that of the proposed CMOEA-MS, respectively.

\section{Experimental Results on Benchmark Suites}

1) Comparisons on LIR-CMOP Suite: The 14 LIR-CMOP problems are challenging for most existing CMOEAs since they have small feasible regions, where some Pareto fronts are obstructed by very large infeasible regions, some Pareto fronts consist of a few disjoint segments or sparse dots obstructed by large infeasible regions, and some feasible regions are even just a curve. As can be seen from the experimental results shown in Table I, both PPS and CMOEA-MS exhibit the best performance on six CMOPs, C-TAEA exhibits the best performance on two CMOPs, while the other three CMOEAs do not gain any best result. In particular, the proposed CMOEA-MS exhibits the best performance on LIR-CMOP5-LIR-CMOP8 and good performance on LIR-CMOP9-LIR-CMOP12, since CMOEA-MS is good at handling the feasible regions that are obstructed by large infeasible regions (LIR-CMOP5-LIR-CMOP8) or disconnected (LIR-CMOP9-LIR-CMOP12). By contrast, 
TABLE II

Statistical Results of IGD ObTAined by TIGE-2, NSGA-II-CDP, C-TAEA, PPS, ToP, AND the Proposed CMOEA-MS ON THE DAS-CMOP BENCHMARK SUite. BEST RESUlt IN EACH ROW IS Highlighted.

\begin{tabular}{|c|c|c|c|c|c|c|}
\hline Problem & TiGE-2 & NSGA-II-CDP & C-TAEA & PPS & ToP & CMOEA-MS \\
\hline DAS-CMOP1 & $1.0259 \mathrm{e}-1(9.94 \mathrm{e}-2) \approx$ & $1.6298 \mathrm{e}-1(2.54 \mathrm{e}-1) \approx$ & $1.0091 \mathrm{e}-2(1.83 \mathrm{e}-3)+$ & $2.0439 \mathrm{e}-3(1.91 \mathrm{e}-4)+$ & $4.9550 \mathrm{e}-2(1.64 \mathrm{e}-1)+$ & $1.4453 \mathrm{e}-1(2.15 \mathrm{e}-1)$ \\
\hline DAS-CMOP2 & $2.5125 \mathrm{e}-2(3.71 \mathrm{e}-3)-$ & $4.8685 \mathrm{e}-2(3.64 \mathrm{e}-2)-$ & $7.8570 \mathrm{e}-3(8.70 \mathrm{e}-4) \approx$ & $4.0043 \mathrm{e}-3(1.99 \mathrm{e}-4) \approx$ & $3.8294 \mathrm{e}-3(8.81 \mathrm{e}-5) \approx$ & $1.6372 \mathrm{e}-2(1.45 \mathrm{e}-2)$ \\
\hline DAS-CMOP3 & $9.8960 \mathrm{e}-2(6.08 \mathrm{e}-2) \approx$ & $1.7765 \mathrm{e}-1(5.93 \mathrm{e}-2) \approx$ & $3.0807 \mathrm{e}-2(9.67 \mathrm{e}-3)+$ & $1.9309 e-2(9.12 e-5)+$ & $8.4918 \mathrm{e}-2(8.66 \mathrm{e}-2) \approx$ & $1.2938 \mathrm{e}-1(4.95 \mathrm{e}-2)$ \\
\hline DAS-CMOP4 & $1.5949 \mathrm{e}-1(2.23 \mathrm{e}-1)-$ & $9.6496 \mathrm{e}-2(2.22 \mathrm{e}-1)-$ & $1.1141 \mathrm{e}-2(2.45 \mathrm{e}-3)+$ & $1.7224 \mathrm{e}-3(8.27 \mathrm{e}-5)+$ & $6.6905 \mathrm{e}-1(2.59 \mathrm{e}-1)-$ & $2.3620 \mathrm{e}-2(7.79 \mathrm{e}-2)$ \\
\hline DAS-CMOP5 & $2.2732 \mathrm{e}-2(4.14 \mathrm{e}-3)-$ & $3.4816 \mathrm{e}-3(7.20 \mathrm{e}-5)-$ & $7.4499 \mathrm{e}-3(4.44 \mathrm{e}-4)-$ & $4.2145 \mathrm{e}-3(2.87 \mathrm{e}-4)-$ & $5.0464 \mathrm{e}-1(2.57 \mathrm{e}-1)-$ & $2.7513 e-3(6.05 e-5)$ \\
\hline DAS-CMOP6 & $4.7244 \mathrm{e}-2(3.93 \mathrm{e}-2) \approx$ & $1.5596 \mathrm{e}-2(3.44 \mathrm{e}-3)+$ & $2.9464 \mathrm{e}-2(6.73 \mathrm{e}-3)-$ & $2.4358 \mathrm{e}-2(1.11 \mathrm{e}-2) \approx$ & $6.4312 \mathrm{e}-1(1.48 \mathrm{e}-1)-$ & $2.6886 \mathrm{e}-2(2.25 \mathrm{e}-2)$ \\
\hline DAS-CMOP7 & $5.6453 \mathrm{e}-2(3.92 \mathrm{e}-3)-$ & $2.3213 e-2(6.85 e-4)-$ & $2.9120 \mathrm{e}-2(5.11 \mathrm{e}-4)-$ & $3.9329 \mathrm{e}-2(5.91 \mathrm{e}-3)-$ & $6.2011 \mathrm{e}-1(2.68 \mathrm{e}-1)-$ & $1.7718 \mathrm{e}-2(2.46 \mathrm{e}-4)$ \\
\hline DAS-CMOP8 & $6.8560 \mathrm{e}-2(3.52 \mathrm{e}-3)-$ & $2.8860 \mathrm{e}-2(1.16 \mathrm{e}-3)-$ & $4.4515 \mathrm{e}-2(2.10 \mathrm{e}-3)-$ & $6.8281 \mathrm{e}-2(1.27 \mathrm{e}-2)-$ & $7.9786 \mathrm{e}-1(1.41 \mathrm{e}-1)-$ & $2.3613 e-2(8.48 e-4)$ \\
\hline DAS-CMOP9 & $6.1238 \mathrm{e}-2(3.14 \mathrm{e}-3)-$ & $3.1116 \mathrm{e}-2(1.68 \mathrm{e}-3)-$ & $4.6351 \mathrm{e}-2(1.65 \mathrm{e}-3)-$ & $3.2196 \mathrm{e}-2(1.80 \mathrm{e}-3)-$ & $2.8120 \mathrm{e}-2(8.33 \mathrm{e}-4)-$ & $2.3157 \mathrm{e}-2(3.83 \mathrm{e}-4)$ \\
\hline$+/-/ \approx$ & $0 / 6 / 3$ & $1 / 6 / 2$ & $3 / 5 / 1$ & $3 / 4 / 2$ & $1 / 6 / 2$ & \\
\hline
\end{tabular}
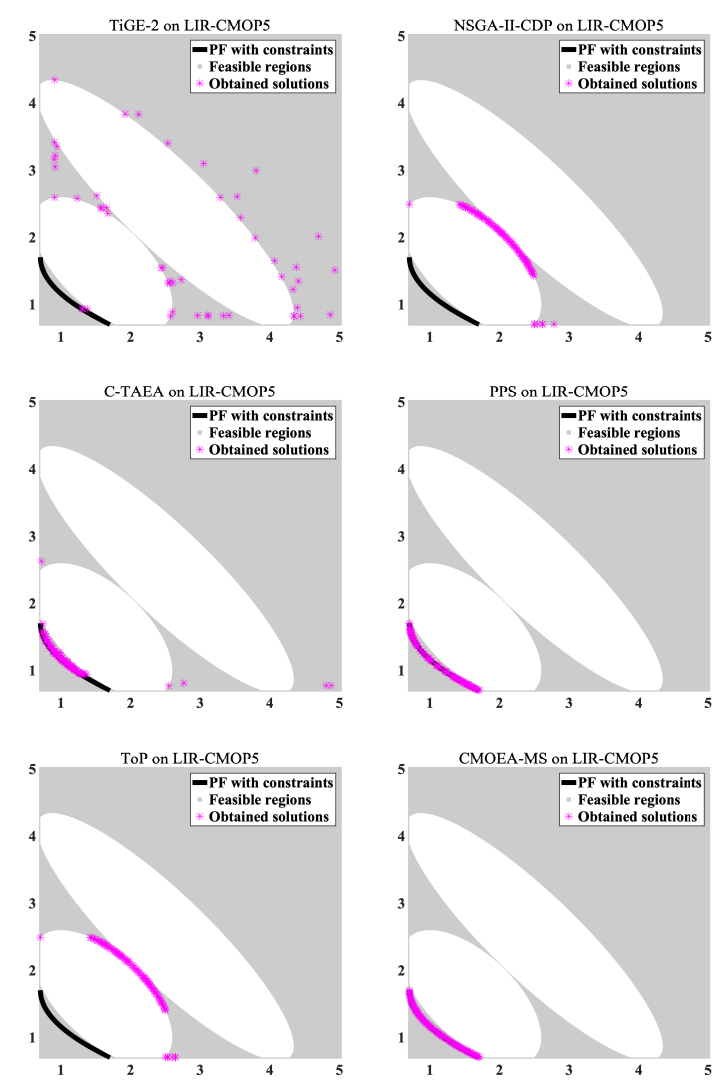

Fig. 9. Populations with the median IGD obtained by TiGE-2, NSGAII-CDP, C-TAEA, PPS, ToP, and the proposed CMOEA-MS on LIRCMOP5.

CMOEA-MS is underperformed by PPS on LIR-CMOP1LIR-CMOP4, whose feasible regions are just curves and difficult to be found by most CMOEAs.

Fig. 9 draws the populations with the median IGD obtained by the six CMOEAs on LIR-CMOP5, whose Pareto front is obstructed by two large infeasible regions. It can be seen that the populations of TiGE-2, NSGA-II$\mathrm{CDP}$, and ToP get stuck in the two infeasible regions and cannot reach the Pareto front, since these three CMOEAs give the objectives a lower priority than the constraints that disable the population from crossing the infeasible region. Although the convergence-oriented archive of C-TAEA also gives the objectives a low priority, the diversity-oriented archive of C-TAEA gives the objectives a higher priority than the constraints that can generate some offspring solutions crossing the infeasible region. On the contrary, PPS and the proposed CMOEAMS can always give the objectives an equal or higher priority than the constraints, and all the solutions obtained by them can cross the infeasible region and reach the Pareto front.

2) Comparisons on DAS-CMOP Suite: The Pareto fronts of DAS-CMOP problems also consist of some disjoint segments obstructed by large infeasible regions; in addition, the feasible regions of some DAS-CMOP problems are far from the unconstrained Pareto front. According to the experimental results given in Table II, the proposed CMOEA-MS also exhibits a very promising performance, having achieved the best results on 4 CMOPs and followed by PPS, NSGA-II-CDP, and ToP. Similar to the performance on LIR-CMOP suite, CMOEA-MS exhibits good performance on DAS-CMOP2, DAS-CMOP5, and DAS-CMOP7-DAS-CMOP9 since they have multiple disconnected feasible regions, while it performs badly on DAS-CMOP1, DAS-CMOP3, DAS-CMOP4, and DASCMOP6 since their feasible regions are very small and easy to be missed. Therefore, the proposed CMOEAMS is good at handling CMOPs with complex feasible regions (e.g., the feasible regions are disconnected, obstructed by large infeasible regions, or disjoint from the unconstrained Pareto front); however, its performance deteriorates when the feasible regions become small.

Fig. 10 depicts the populations with the median IGD obtained by the six CMOEAs on DAS-CMOP8, whose feasible regions are disconnected and far from the unconstrained Pareto front $f_{1}^{2}+f_{2}^{2}+f_{3}^{2}=1$. Obviously, TiGE-2, NSGA-II-CDP, and C-TAEA can find a set of solutions lying on the feasible regions. By contrast, PPS can only find a few feasible solutions due to the high priority of the objectives. ToP can hardly find some solutions on the feasible regions since its reproduction strategy is tailored for the CMOPs with constraints in both decision and objective spaces and is ineffective for DAS-CMOP8 with a multi-modal landscape, though it uses the same constraint handling technique to NSGA-II-CDP. Owing to the lower priority of the objectives given in Stage B, the proposed CMOEA-MS can find a large number of uniformly distributed feasible solutions.

3) Comparisons on MW Suite: MW is a more generic test suite that the feasible regions have diverse characteristics, where some feasible regions are disconnected, 
TABLE III

StATistical Results of IGD Obtained by TiGE-2, NSGA-II-CDP, C-TAEA, PPS, ToP, AND the Proposed CMOEA-MS on THE MW Benchmark Suite. Best Result in Each Row is Highlighted.

\begin{tabular}{|c|c|c|c|c|c|c|}
\hline Problem & TiGE-2 & NSGA-II-CDP & C-TAEA & PPS & ToP & CMOEA-MS \\
\hline MW1 & $2.3382 \mathrm{e}-1(2.37 \mathrm{e}-1)-$ & $2.0813 e-3(7.94 e-5)-$ & $2.0137 \mathrm{e}-3(8.76 \mathrm{e}-5)-$ & $2.6688 \mathrm{e}-3(8.83 e-5)-$ & $1.3725 \mathrm{e}-1(2.14 \mathrm{e}-1)-$ & $1.6155 \mathrm{e}-3(1.04 \mathrm{e}-5)$ \\
\hline MW2 & $6.4655 \mathrm{e}-1(9.36 \mathrm{e}-2)-$ & $1.7289 \mathrm{e}-2(1.19 \mathrm{e}-2)-$ & $1.0135 \mathrm{e}-2(7.03 \mathrm{e}-3) \approx$ & $7.3785 \mathrm{e}-2(4.82 \mathrm{e}-2)-$ & $9.1757 \mathrm{e}-2(7.72 \mathrm{e}-2)-$ & $8.8565 e-3(5.37 e-3)$ \\
\hline MW3 & $2.4217 \mathrm{e}-2(3.38 \mathrm{e}-3)-$ & $5.6382 \mathrm{e}-3(1.95 \mathrm{e}-4)-$ & $4.5625 \mathrm{e}-3(1.65 \mathrm{e}-4) \approx$ & $6.6556 \mathrm{e}-3(7.44 \mathrm{e}-4)-$ & $4.6446 \mathrm{e}-1(4.75 \mathrm{e}-1)-$ & $4.5349 \mathrm{e}-3(1.21 \mathrm{e}-4)$ \\
\hline MW4 & $4.2608 \mathrm{e}-2(1.60 \mathrm{e}-3)-$ & $3.1808 \mathrm{e}-2(1.21 \mathrm{e}-3)-$ & $2.3389 e-2(9.64 e-5)-$ & $3.1116 \mathrm{e}-2(8.85 \mathrm{e}-4)-$ & $9.2925 \mathrm{e}-2(1.47 \mathrm{e}-1)-$ & $2.2720 \mathrm{e}-2(1.47 \mathrm{e}-4)$ \\
\hline MW5 & $4.1708 \mathrm{e}-2(3.57 \mathrm{e}-2)-$ & $6.8267 \mathrm{e}-2(2.12 \mathrm{e}-1) \approx$ & $8.2972 \mathrm{e}-3(1.77 \mathrm{e}-3) \approx$ & $1.2808 \mathrm{e}-1(2.86 \mathrm{e}-1) \approx$ & $5.9780 \mathrm{e}-1(3.00 \mathrm{e}-1)-$ & $6.4659 \mathrm{e}-3(5.64 \mathrm{e}-3)$ \\
\hline MW6 & $6.3976 \mathrm{e}-1(4.28 \mathrm{e}-1)-$ & $1.5742 \mathrm{e}-2(9.93 \mathrm{e}-3) \approx$ & $5.4619 \mathrm{e}-3(3.12 \mathrm{e}-3) \approx$ & $3.0476 \mathrm{e}-1(2.64 \mathrm{e}-1)-$ & $2.5613 \mathrm{e}-1(2.51 \mathrm{e}-1)-$ & $1.5769 \mathrm{e}-2(8.90 \mathrm{e}-3)$ \\
\hline MW7 & $4.0485 \mathrm{e}-2(2.13 \mathrm{e}-2)-$ & $4.1938 \mathrm{e}-2(1.29 \mathrm{e}-1)-$ & $6.3718 \mathrm{e}-3(4.49 \mathrm{e}-4)-$ & $5.3326 \mathrm{e}-3(4.30 \mathrm{e}-4)-$ & $1.0465 \mathrm{e}-1(1.79 \mathrm{e}-1)-$ & $3.8232 \mathrm{e}-3(6.91 \mathrm{e}-5)$ \\
\hline MW8 & $8.9002 \mathrm{e}-1(8.47 \mathrm{e}-2)-$ & $2.9628 \mathrm{e}-2(1.10 \mathrm{e}-3)-$ & $2.5459 \mathrm{e}-2(3.03 \mathrm{e}-4)-$ & $5.6767 \mathrm{e}-2(1.49 \mathrm{e}-2)-$ & $6.8136 \mathrm{e}-2(1.81 \mathrm{e}-2)-$ & $2.2932 \mathrm{e}-2(8.27 \mathrm{e}-4)$ \\
\hline MW9 & $1.0798 \mathrm{e}-1(9.00 \mathrm{e}-2)-$ & $4.9567 \mathrm{e}-3(2.31 \mathrm{e}-4)-$ & $9.9748 \mathrm{e}-3(6.98 \mathrm{e}-4)-$ & $6.5818 \mathrm{e}-3(5.30 \mathrm{e}-4)-$ & $5.7108 \mathrm{e}-3(3.20 \mathrm{e}-4)-$ & $4.0820 \mathrm{e}-3(8.42 \mathrm{e}-5)$ \\
\hline MW10 & $2.4598 \mathrm{e}-2(1.58 \mathrm{e}-2)-$ & $9.7869 \mathrm{e}-2(5.59 \mathrm{e}-2)-$ & $8.6793 \mathrm{e}-3(5.45 \mathrm{e}-3) \approx$ & $2.2177 \mathrm{e}-1(1.23 \mathrm{e}-1)-$ & $3.9603 \mathrm{e}-1(3.58 \mathrm{e}-1)-$ & $1.2972 \mathrm{e}-2(1.92 \mathrm{e}-2)$ \\
\hline MW11 & $3.1339 \mathrm{e}-2(4.84 \mathrm{e}-3)-$ & $6.8677 \mathrm{e}-3(3.20 \mathrm{e}-4)-$ & $1.1922 \mathrm{e}-2(1.22 \mathrm{e}-3)-$ & $7.4482 \mathrm{e}-3(4.75 \mathrm{e}-4)-$ & $7.3637 \mathrm{e}-2(1.26 \mathrm{e}-1)-$ & $5.8206 e-3(9.06 e-5)$ \\
\hline MW12 & $2.9776 \mathrm{e}-2(8.05 \mathrm{e}-3)-$ & $5.5490 \mathrm{e}-3(1.88 \mathrm{e}-4)-$ & $7.6746 \mathrm{e}-3(4.82 \mathrm{e}-4)-$ & $7.0151 \mathrm{e}-3(6.69 \mathrm{e}-4)-$ & $2.8050 \mathrm{e}-1(3.41 \mathrm{e}-1)-$ & $4.6382 \mathrm{e}-3(1.02 \mathrm{e}-4)$ \\
\hline MW13 & $2.3000 \mathrm{e}+0(2.03 \mathrm{e}-2)-$ & $7.9294 \mathrm{e}-2(1.13 \mathrm{e}-1)-$ & $1.9117 \mathrm{e}-2(8.21 \mathrm{e}-3) \approx$ & $1.7994 \mathrm{e}-1(1.25 \mathrm{e}-1)-$ & $2.5678 \mathrm{e}-1(2.29 \mathrm{e}-1)-$ & $2.6401 \mathrm{e}-2(1.19 \mathrm{e}-2)$ \\
\hline MW14 & $9.0982 \mathrm{e}-2(4.17 \mathrm{e}-3)-$ & $7.1194 \mathrm{e}-2(3.41 \mathrm{e}-3)-$ & $5.4800 \mathrm{e}-2(6.26 \mathrm{e}-4)-$ & $1.1287 \mathrm{e}-1(1.28 \mathrm{e}-2)-$ & $8.6473 e-2(7.19 e-3)-$ & $5.3318 \mathrm{e}-2(5.80 \mathrm{e}-4)$ \\
\hline$+1-1 \approx$ & $0 / 14 / 0$ & $0 / 12 / 2$ & & $0 / 13 / 1$ & $0 / 14 / 0$ & \\
\hline
\end{tabular}
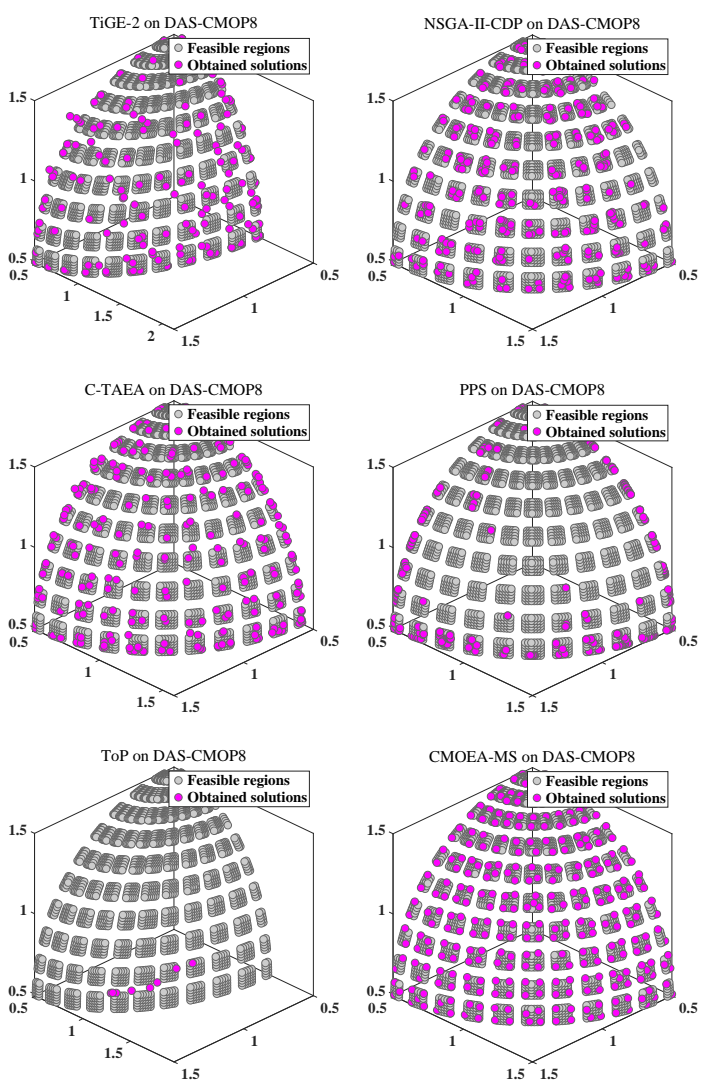

Fig. 10. Populations with the median IGD obtained by TiGE-2, NSGAII-CDP, C-TAEA, PPS, ToP, and the proposed CMOEA-MS on DASCMOP8.

some feasible regions are separated by large infeasible regions, and some others are far from the unconstrained Pareto front. Table III lists the IGD results of the six compared CMOEAs on MW1-MW14. As can be seen, CMOEA-MS exhibits the best overall performance on the MW suite, which gains the best results on nine CMOPs and competitive results on the other five CMOPs. This is because the feasible regions of MW suite are relatively large and can be easily handled.

Fig. 11 plots the populations with the median IGD obtained by the six CMOEAs on MW14. The feasible region of MW14 is narrow but involves the whole
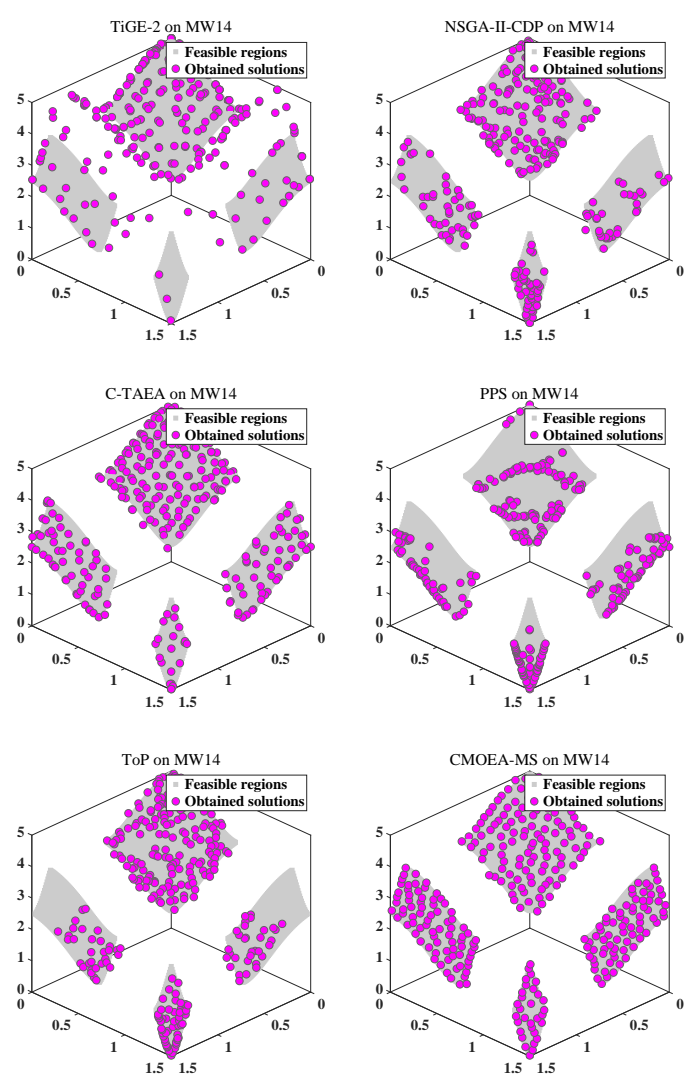

Fig. 11. Populations with the median IGD obtained by TiGE-2, NSGAII-CDP, C-TAEA, PPS, ToP, and the proposed CMOEA-MS on MW14.

unconstrained Pareto front; that is, it can be handled by giving any priority to the objectives, even without considering the constraints. As shown in Fig. 11, all the six CMOEAs can obtain a number of feasible solutions on the Pareto front. While in terms of diversity, the populations obtained by C-TAEA and CMOEA-MS are better than those obtained by TiGE-2, NSGA-II-CDP, PPS, and ToP. Hence, the proposed CMOEA-MS is also competitive for tackling simple feasible regions.

4) Comparisons on DC-DTLZ Suite: The DC-DTLZ problems have complex constraints in the decision space and highly multi-modal landscapes, which pose chal- 
TABLE IV

Statistical Results of IGD ObTAined by TIGE-2, NSGA-II-CDP, C-TAEA, PPS, ToP, AND the Proposed CMOEA-MS ON THE DC-DTLZ BenCHMARK Suite. Best Result in EACH Row is Highlighted.

\begin{tabular}{|c|c|c|c|c|c|c|}
\hline Problem & TiGE-2 & NSGA-II-CDP & C-TAEA & PPS & ToP & CMOEA-MS \\
\hline DC1-DTLZ1 & $1.5050 \mathrm{e}+0(8.33 \mathrm{e}-1)-$ & $8.7857 \mathrm{e}-3(3.61 \mathrm{e}-4)-$ & $7.3792 \mathrm{e}-3(6.36 \mathrm{e}-5)-$ & $1.1772 \mathrm{e}-2(3.43 \mathrm{e}-4)-$ & $1.4641 \mathrm{e}-1(1.56 \mathrm{e}-1)-$ & $6.2527 e-3(2.93 e-5)$ \\
\hline DC1-DTLZ3 & $9.5954 \mathrm{e}-1(1.79 \mathrm{e}-1)-$ & $1.1490 \mathrm{e}-1(1.89 \mathrm{e}-3)-$ & $1.0786 \mathrm{e}-1(9.47 \mathrm{e}-4)-$ & $1.1131 \mathrm{e}-1(1.22 \mathrm{e}-3)-$ & $1.0055 \mathrm{e}+0(3.89 \mathrm{e}-1)-$ & $1.0506 \mathrm{e}-1(3.83 \mathrm{e}-4)$ \\
\hline DC2-DTLZ1 & $2.9702 \mathrm{e}-1(5.32 \mathrm{e}-2)-$ & $\mathrm{N} / \mathrm{A}$ & $1.1669 \mathrm{e}-2(3.03 \mathrm{e}-5)-$ & $1.5667 \mathrm{e}-2(2.50 \mathrm{e}-4)-$ & $\mathrm{N} / \mathrm{A}$ & $1.1161 \mathrm{e}-2(4.84 \mathrm{e}-5)$ \\
\hline DC2-DTLZ3 & $3.5448 \mathrm{e}-1(1.82 \mathrm{e}-1)-$ & $5.6046 \mathrm{e}-1(9.78 \mathrm{e}-4)-$ & $3.1278 \mathrm{e}-2(1.79 \mathrm{e}-4)-$ & $4.0098 \mathrm{e}-2(1.15 \mathrm{e}-3)-$ & $\mathrm{N} / \mathrm{A}$ & $2.9734 \mathrm{e}-2(1.29 \mathrm{e}-4)$ \\
\hline DC3-DTLZ1 & $3.0928 \mathrm{e}+0(1.42 \mathrm{e}+0)-$ & $1.3812 \mathrm{e}-1(8.26 \mathrm{e}-2)-$ & $4.6615 e-3(5.28 \mathrm{e}-5)+$ & $8.2808 \mathrm{e}-3(5.25 \mathrm{e}-4)+$ & $6.8533 \mathrm{e}+0(1.44 \mathrm{e}+0)-$ & $1.9386 \mathrm{e}-2(5.58 \mathrm{e}-3)$ \\
\hline DC3-DTLZ3 & $1.6413 \mathrm{e}+0(3.33 \mathrm{e}-1)-$ & $6.6709 \mathrm{e}-1(1.99 \mathrm{e}-1)-$ & $1.5867 \mathrm{e}-1(1.61 \mathrm{e}-3)+$ & $1.5906 \mathrm{e}-1(9.43 \mathrm{e}-4)+$ & $2.6436 \mathrm{e}+0(4.39 \mathrm{e}-1)-$ & $2.2425 \mathrm{e}-1(4.74 \mathrm{e}-2)$ \\
\hline$+/-1 \approx$ & $0 / 6 / 0$ & $0 / 5 / 0$ & $2 / 4 / 0$ & $2 / 4 / 0$ & $0 / 4 / 0$ & \\
\hline
\end{tabular}

lenges for CMOEAs to converge to the constrained Pareto front. Table IV presents the IGD values of the six compared MOEAs on the six DC-DTLZ problems, where 'N/A' indicates that no feasible solution is found by the CMOEA. It can be observed that the proposed CMOEAMS obtains four best results and C-TAEA obtains two best results. In terms of statistic test, CMOEA-MS significantly outperforms the compared CMOEAs on four to six CMOPs, which indicates that CMOEA-MS is also promising for solving the DC-DTLZ suite.

Since the population with slightly worse diversity may have the best IGD value due to the biased distribution of the reference points [49], the HV results of the six compared MOEAs on all the benchmark CMOPs are listed in Supplementary Materials II. It can be found from the statistical results that the proposed CMOEAMS also obtains the best results on most CMOPs.

\section{E. Effectiveness of the Two-Stage Framework of CMOEA-MS}

This subsection performs ablation study to verify the effectiveness of the core components of the proposed CMOEA-MS, where CMOEA-MS is compared to its three variants on the MW suite. The first variant CMOEA$\mathrm{MS}^{1}$ replaces the first objective $S D E(\mathbf{x})$ in Stage A by the sum of all the objectives, which is used to verify the effectiveness of the two new objectives proposed for Stage A. The second variant CMOEA-MS ${ }^{2}$ and the third variant CMOEA-MS ${ }^{3}$ always uses the fitness evaluation strategy of Stage A and Stage B, respectively, which are used to verify the effectiveness of using both the two fitness evaluation strategies (i.e., different priorities of objectives and constraints) simultaneously.

Table V presents the IGD results of CMOEA-MS and its three variants on MW1-MW14. It can be found that CMOEA-MS is statistically similar to or significantly better than CMOEA-MS ${ }^{1}$ on all the CMOPs, which verifies the effectiveness of the first objective $S D E(\mathbf{x})$. Besides, CMOEA-MS is also statistically similar to or significantly better than CMOEA-MS ${ }^{2}$ and CMOEA-MS ${ }^{3}$, hence the effectiveness of different priorities of objectives and constraints in the two stages can be verified.

\section{F. Experimental Results on Real-World CMOPs}

Lastly, the performance of CMOEA-MS is verified on three CMOPs from real-world applications, i.e., the car side impact problem [22], the vibration platform
TABLE V

STATISTICAL RESULTS OF IGD OBTAINED BY CMOEA-MS AND ITS THREE VARIANTS ON THE MW BENCHMARK SUITE. BEST RESUlt IN EACH ROW IS HighLIGHTED.

\begin{tabular}{|c|c|c|c|c|}
\hline Problem & CMOEA-N & CMOEA-MS $^{1}$ & CMOEA-MS $^{2}$ & CMOEA-MS ${ }^{3}$ \\
\hline MW1 & 1.61 & $6191 \mathrm{e}-3 \approx$ & $1.8077 \mathrm{e}-2-$ & $1.6183 \mathrm{e}-3 \approx$ \\
\hline MW2 & & & $2-$ & $2-$ \\
\hline & $49 e-3$ & $2.7841 \mathrm{e}-1$ - & $6.3663 e-2-$ & $-2 \approx$ \\
\hline MW4 & De-2 & $2.2730 \mathrm{e}-2 \approx$ & $5.5664 \mathrm{e}-2-$ & $2.2758 \mathrm{e}-2 \approx$ \\
\hline MW5 & -3 & -1 - & $9.5492 \mathrm{e}-2-$ & $2.1545 \mathrm{e}-2 \approx$ \\
\hline MW6 & -2 & $-1-$ & $-2-$ & $-2-$ \\
\hline MW7 & & & $-1-$ & $-3 \approx$ \\
\hline & & & $-2-$ & $-2 \approx$ \\
\hline & & $1-$ & $-1-$ & e-3 $\approx$ \\
\hline MW1 & & $1-$ & $-2-$ & e-2 - \\
\hline & & $1-$ & $-1-$ & $\mathrm{e}-3 \approx$ \\
\hline & & $1-$ & e-1 - & $2 \mathrm{e}-3 \approx$ \\
\hline & & 4.75 & $2.2163 e-1-$ & $1.3213 \mathrm{e}-1-$ \\
\hline & & $5.3481 \mathrm{e}-2 \approx$ & $1.1589 \mathrm{e}-1-$ & $5.3783 \mathrm{e}-2 \approx$ \\
\hline & & $0 / 8 / 6$ & $0 / 14 / 0$ & $0 / 4 / 10$ \\
\hline
\end{tabular}

CMOEA-MS $^{1}$ replaces the first objective $S D E$ in Stage A by the sum of all the objectives; CMOEA-MS ${ }^{2}$ always uses the fitness evaluation strategy of Stage A; CMOEA-MS ${ }^{3}$ always uses the fitness evaluation strategy of Stage B.

design problem [15], and the water resource problem [50]. The car side impact problem aims to minimize the weight of the car, the pubic force of passenger, and the average velocity of V-pillar, and the solutions should satisfy the limiting values of ten aspects. The vibration platform design problem aims to maximize the fundamental frequency and minimize the cost, and the solutions should meet the limiting values of mass and width of layers. The water resource problem aims to minimize four costs and the expected economic loss, and the solutions are restricted by the constraints of yearly statistical performance. The mathematical definitions of these CMOPs can be found in their original literatures.

The parameters in the three CMOPs as well as the HV results of six CMOEAs are presented in Table VI. As can be observed from the table, the proposed CMOEAMS outperforms the other five CMOEAs on the car side impact problem and the vibration platform design problem, while it is slightly worse than PPS on the water resource problem. Besides, Fig. 12 shows the populations with the median HV obtained by the six CMOEAs on the car side impact problem. It can be found that the population diversity of CMOEA-MS is similar to ToP and much better than TiGE-2, NSGA-II-CDP, C-TAEA, and PPS. In short, the three real-world CMOPs are not difficult and the proposed CMOEA-MS shows a slightly 
TABLE VI

Statistical Results of HV ObTAined by TiGE-2, NSGA-II-CDP, C-TAEA, PPS, ToP, AND the Proposed CMOEA-MS ON Three REAL-WORLD CMOPs. BEST RESULT IN EACH ROW IS Highlighted.

\begin{tabular}{|c|c|c|c|c|c|c|}
\hline $\begin{array}{c}\text { Problem } \\
\text { (no. of variables / } \\
\text { no. of objectives / } \\
\text { no. of constraints) }\end{array}$ & TiGE-2 & NSGA-II-CDP & C-TAEA & PPS & $\mathrm{ToP}$ & CMOEA-MS \\
\hline $\begin{array}{c}\text { Car side } \\
\text { impact problem } \\
(7 / 3 / 10)\end{array}$ & $2.2297 \mathrm{e}-2(5.55 \mathrm{e}-3)-$ & $3.6671 \mathrm{e}-2(3.88 \mathrm{e}-5)-$ & $3.6883 e-2(2.17 e-5)-$ & $3.6095 \mathrm{e}-2(1.84 \mathrm{e}-4)-$ & $3.6787 \mathrm{e}-2(4.12 \mathrm{e}-5)-$ & $3.7056 \mathrm{e}-2(2.78 \mathrm{e}-5)$ \\
\hline $\begin{array}{l}\text { Vibration platform } \\
\text { design problem } \\
(5 / 2 / 5)\end{array}$ & $8.2110 \mathrm{e}-1(1.24 \mathrm{e}-1)-$ & $8.4142 \mathrm{e}-1(8.48 \mathrm{e}-2) \approx$ & $6.6036 \mathrm{e}-1(1.62 \mathrm{e}-1)-$ & $7.2492 \mathrm{e}-1(1.58 \mathrm{e}-1)-$ & $7.4261 \mathrm{e}-1(1.73 \mathrm{e}-1) \approx$ & $9.2627 \mathrm{e}-1(6.60 \mathrm{e}-2)$ \\
\hline $\begin{array}{c}\text { Water resource } \\
\text { problem } \\
(3 / 5 / 7)\end{array}$ & $1.6611 \mathrm{e}-1(2.31 \mathrm{e}-4)+$ & $1.6700 \mathrm{e}-1(4.07 \mathrm{e}-4)+$ & $1.6650 \mathrm{e}-1(3.60 \mathrm{e}-4)+$ & $1.6894 \mathrm{e}-1(1.82 \mathrm{e}-4)+$ & $1.6546 \mathrm{e}-1(5.49 \mathrm{e}-4) \approx$ & $1.6535 \mathrm{e}-1(3.35 \mathrm{e}-4)$ \\
\hline$+/-1 \approx$ & $1 / 2 / 0$ & $1 / 1 / 1$ & $1 / 2 / 0$ & $1 / 2 / 0$ & $0 / 1 / 2$ & \\
\hline
\end{tabular}
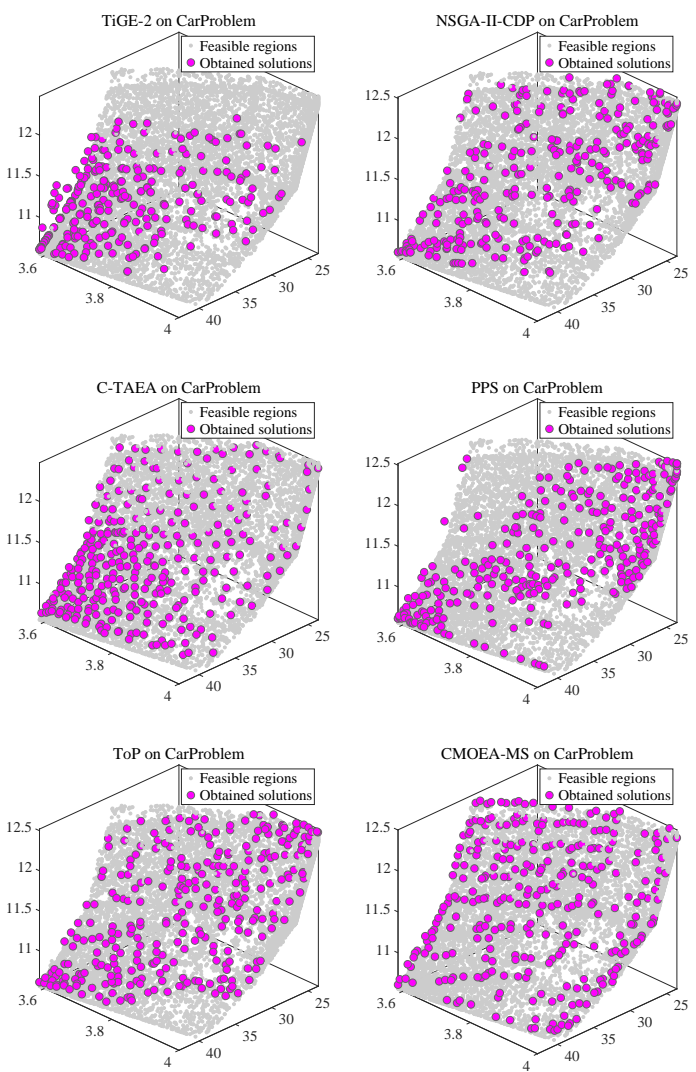

Fig. 12. Populations with the median HV obtained by TiGE-2, NSGAII-CDP, C-TAEA, PPS, ToP, and the proposed CMOEA-MS on the car side impact problem.

better performance than the compared CMOEAs.

\section{CONCLUSIONS}

In this paper, we have proposed a two-stage CMOEA for solving CMOPs with different feasible regions. The core idea of the proposed algorithm is to adaptively balance the objective optimization and constraint satisfaction during the evolutionary process, where the proposed algorithm enters one of the two stages according to the feasibility of the current population at each generation. The Stage A indicates that most solutions are infeasible and gives the objective an equal priority to the constraints, so that some infeasible solutions can be maintained to help the population cross infeasible regions. The Stage B indicates that most solutions are feasible and gives the objectives a lower priority to the constraints, so that more feasible solutions can be found to help the population spread along the feasible boundaries. By using different priorities of objectives and constraints in the two stages, the objective optimization and constraint satisfaction can be well balanced when tackling different types of feasible regions.

In the experiments, the proposed algorithm has been compared to five popular CMOEAs on 43 benchmark CMOPs from four recently proposed test suites. The statistical results have indicated that the proposed algorithm has a better overall performance than the compared CMOEAs on the 43 CMOPs with different feasible regions. Besides, the performance of the proposed algorithm has also been verified on three real-world CMOPs.

By revealing the limitations of existing CMOEAs and proposing a new multi-stage framework, this work has confirmed the importance of balancing objective optimization and constraint satisfaction in solving CMOPs. Although the proposed algorithm has shown promising performance in the experiments, it is desirable to extend the proposed multi-stage framework for solving other more challenging CMOPs. For example, the proposed framework can be embedded with more effective environmental selection strategies for solving constrained many-objective optimization problems (i.e., the UAV route-planning problem [51]), and it can also be embedded with other reproduction operators for solving largescale CMOPs (i.e., time-varying ratio error estimation [1]). Besides, it is reasonable to improve the performance of the proposed framework on CMOPs with very small feasible regions such as LIR-CMOP1-LIR-CMOP4.

\section{REFERENCES}

[1] C. He, R. Cheng, C. Zhang, Y. Tian, H. Li, and X. Yao, "Multiobjective formulation and analysis of the time-varying ratio error estimation," IEEE Transactions on Evolutionary Computation, 2020, in press.

[2] R. Datta, S. Pradhan, and B. Bhattacharya, "Analysis and design optimization of a robotic gripper using multiobjective genetic algorithm," IEEE Transactions on Systems Man and Cybernetics Systems, vol. 46, no. 1, pp. 16-26, 2015.

[3] X. Zhang, Y. Tian, R. Cheng, and Y. Jin, "An efficient approach to non-dominated sorting for evolutionary multi-objective optimization," IEEE Transactions on Evolutionary Computation, vol. 19, no. 2, pp. 201-213, 2015. 
[4] Y. Tian, C. He, R. Cheng, and X. Zhang, "A multistage evolutionary algorithm for better diversity preservation in multiobjective optimization," IEEE Transactions on Systems, Man, and Cybernetics: Systems, 2019, in press.

[5] Y. Tian, X. Xiang, X. Zhang, R. Cheng, and Y. Jin, "Sampling reference points on the Pareto fronts of benchmark multi-objective optimization problems," in Proceedings of the 2018 IEEE Congress on Evolutionary Computation, 2018.

[6] Q. Zhang and H. Li, "MOEA/D: A multiobjective evolutionary algorithm based on decomposition," IEEE Transactions on Evolutionary Computation, vol. 11, no. 6, pp. 712-731, 2007.

[7] J. Bader and E. Zitzler, "HypE: an algorithm for fast hypervolumebased many-objective optimization," Evolutionary Computation, vol. 19, no. 1, pp. 45-76, 2011.

[8] Y. Tian, R. Cheng, X. Zhang, F. Cheng, and Y. Jin, "An indicator based multi-objective evolutionary algorithm with reference point adaptation for better versatility," IEEE Transactions on Evolutionary Computation, vol. 22, no. 4, pp. 609-622, 2018.

[9] K. Deb, A. Pratap, S. Agarwal, and T. Meyarivan, "A fast and elitist multiobjective genetic algorithm: NSGA-II," IEEE Transactions on Evolutionary Computation, vol. 6, no. 2, pp. 182-197, 2002.

[10] K. Li, R. Chen, G. Fu, and X. Yao, "Two-archive evolutionary algorithm for constrained multi-objective optimization," IEEE Transactions on Evolutionary Computation, vol. 23, no. 2, pp. 303 315, 2018.

[11] Z. Fan, W. Li, X. Cai, L. Hui, and E. D. Goodman, "Push and pull search for solving constrained multi-objective optimization problems," Swarm and Evolutionary Computation, no. 44, pp. 665679,2019

[12] Z. Fan, W. Li, X. Cai, H. Han, F. Yi, Y. You, J. Mo, C. Wei, and E. Goodman, "An improved epsilon constraint-handling method in MOEA/D for CMOPs with large infeasible regions," Soft Computing, vol. 23, pp. 12491-12510, 2019.

[13] Z. Ma and Y. Wang, "Evolutionary constrained multiobjective optimization: Test suite construction and performance comparisons," IEEE Transactions on Evolutionary Computation, vol. 23, no. 6, pp. 972-986, 2019.

[14] Z. Z. Liu and Y. Wang, "Handling constrained multiobjective optimization problems with constraints in both the decision and objective spaces," IEEE Transactions on Evolutionary Computation, vol. 23, no. 5, pp. 870-884, 2019.

[15] T. Ray, K. Tai, and K. C. Seow, "Multiobjective design optimization by an evolutionary algorithm," Engineering Optimization, vol. 33, no. 4, pp. 399-424, 2001.

[16] A. Angantyr, J. Andersson, and J. O. Aidanpaa, "Constrained optimization based on a multiobjective evolutionary algorithm," in Proceeding of IEEE Congress on Evolutionary Computation, 2003.

[17] N. Young, "Blended ranking to cross infeasible regions in constrainedmultiobjective problems," in Proceedings of the 2005 International Conference on Computational Intelligence Modeling, Control and Automation, 2005, pp. 191-196.

[18] Y. G. Woldesenbet, B. G. Tessema, and G. G. Yen, "Constraint handling in multi-objective evolutionary optimization," in IEEE Transactions on Evolutionary Computation, 2007, pp. 514-525.

[19] W. Ning, B. Guo, Y. Yan, X. Wu, J. Wu, and Z. Dan, “Constrained multi-objective optimization using constrained non-dominated sorting combined with an improved hybrid multi-objective evolutionary algorithm," Engineering Optimization, vol. 49, no. 10, pp. $1-20,2017$

[20] Y. Zhou, Z. Min, J. Wang, Z. Zhang, and J. Zhang, "Tri-goal evolution framework for constrained many-objective optimization," IEEE Transactions on Systems Man and Cybernetics Systems, vol. 50, no. 8, pp. 3086-3099, 2020.

[21] C. A. C. Coello and A. D. Christiansen, "MOSES: A multiobjective optimization tool for engineering design," Engineering Optimization, vol. 31, no. 3, pp. 337-368, 1999.

[22] H. Jain and K. Deb, "An evolutionary many-objective optimization algorithm using reference-point based nondominated sorting approach, part II: Handling constraints and extending to an adaptive approach," IEEE Transactions on Evolutionary Computation, vol. 18, no. 4, pp. 602-622, 2014.

[23] R. Cheng, Y. Jin, M. Olhofer, and B. Sendhoff, "A reference vector guided evolutionary algorithm for many-objective optimization," IEEE Transactions on Evolutionary Computation, vol. 20, no. 5, pp. 773-791, Oct 2016.

[24] M. Asafuddoula, T. Ray, and R. Sarker, "A decomposition based evolutionary algorithm for many objective optimization," IEEE
Transactions on Evolutionary Computation, vol. 19, no. 3, pp. 445460, 2015.

[25] T. Takahama and S. Sakai, "Efficient constrained optimization by the $\varepsilon$ constrained adaptive differential evolution," in Proceedings of the 2010 IEEE Congress on Evolutionary Computation, 2010.

[26] Z. Fan, W. Li, X. Cai, K. Hu, H. Lin, and H. Li, "Angle-based constrained dominance principle in MOEA/D for constrained multi-objective optimization problems," in Proceedings of the 2016 IEEE Congress on Evolutionary Computation, 2016, pp. 460-467.

[27] K. Li, K. Deb, Q. Zhang, and S. Kwong, "Combining dominance and decomposition in evolutionary many-objective optimization," IEEE Transactions on Evolutionary Computation, 2015.

[28] A. Oyama, K. Shimoyama, and K. Fujii, "New constraint-handling method for multi-objective and multi-constraint evolutionary optimization," Transactions of the Japan Society for Aeronautical and Space Sciences, vol. 50, no. 167, pp. 56-62, 2007.

[29] S. Kukkonen and J. Lampinen, "GDE3: The third evolution step of generalized differential evolution," in Proceddings of IEEE Congress on Evolutionary Computation, vol. 1, 10 2005, pp. 443 - 450 Vol.1.

[30] A. E. Sorkhabi, M. D. Amiri, and A. R. Khanteymoori, "Duality evolution: an efficient approach to constraint handling in multiobjective particle swarm optimization," Soft Computing, vol. 21, no. 24, pp. 7251-7267, 2017.

[31] Y. Wang and Z. Cai, "A dynamic hybrid framework for constrained evolutionary optimization," IEEE Transactions on Systems Man and Cybernetics, Part B: Cybernetics, vol. 42, no. 1, pp. 203-217, 2012.

[32] E. Kieffer, G. Danoy, P. Bouvry, and A. Nagih, "A new coevolutionary algorithm based on constraint decomposition," in Proceedings of the 2017 IEEE International Parallel and Distributed Processing Symposium Workshops, 2017, pp. 492-500.

[33] W. F. Gao, G. G. Yen, and S. Y. Liu, "A dual-population differential evolution with coevolution for constrained optimization," IEEE Transactions on Cybernetics, vol. 45, no. 5, pp. 1108-1121, 2017.

[34] B. Liu, H. Ma, X. Zhang, and Y. Zhou, "A memetic co-evolutionary differential evolution algorithm for constrained optimization," in Proceedings of the 2007 IEEE Congress on Evolutionary Computation, 2007, pp. 2996-3002.

[35] W. Yong, B. Xu, G. Sun, and S. Yang, "A two-phase differential evolution for uniform designs in constrained experimental domains," IEEE Transactions on Evolutionary Computation, vol. 21, no. 5, pp. 665-680, 2017.

[36] S. Zeng, R. Jiao, C. Li, X. Li, and J. S. Alkasassbeh, "A general framework of dynamic constrained multiobjective evolutionary algorithms for constrained optimization," IEEE Transactions on Cybernetics, vol. 47, no. 9, pp. 2678-2688, 2017.

[37] S. Zeng, R. Jiao, C. Li, and R. Wang, "Constrained optimisation by solving equivalent dynamic loosely-constrained multiobjective optimisation problem," International Journal of Bio-inspired Computation, vol. 13, no. 2, pp. 86-101, 2019.

[38] E. Zitzler, M. Laumanns, and L. Thiele, "SPEA2: Improving the strength Pareto evolutionary algorithm for multiobjective optimization," in Proceedings of the Fifth Conference on Evolutionary Methods for Design, Optimization and Control with Applications to Industrial Problems, 2001, pp. 95-100.

[39] Y. Tian, H. Wang, X. Zhang, and Y. Jin, "Effectiveness and efficiency of non-dominated sorting for evolutionary multiand many-objective optimization," Complex $\mathcal{E}$ Intelligent Systems, vol. 3, no. 4, pp. 247-263, 2017.

[40] Y. Tian, X. Zheng, X. Zhang, and Y. Jin, "Efficient large-scale multiobjective optimization based on a competitive swarm optimizer," IEEE Transactions on Cybernetics, vol. 50, no. 8, pp. 3696-3708, 2020.

[41] Z. Fan, W. Li, X. Cai, L. Hui, and E. D. Goodman, "Difficulty adjustable and scalable constrained multi-objective test problem toolkit," Evolutionary Computation, 2019, in press.

[42] Y. Tian, R. Cheng, X. Zhang, and Y. Jin, "PlatEMO: A MATLAB platform for evolutionary multi-objective optimization [educational forum]," IEEE Computational Intelligence Magazine, vol. 12, no. 4, pp. 73-87, Nov 2017.

[43] K. Deb and R. B. Agrawal, "Simulated binary crossover for continuous search space," Complex Systems, vol. 9, no. 4, pp. 115148, 1995.

[44] K. Deb and M. Goyal, "A combined genetic adaptive search (GeneAS) for engineering design," Computer Science and Informatics, vol. 26, no. 4, pp. 30-45, 1996. 
[45] K. Price, R. M. Storn, and J. A. Lampinen, Differential evolution: A practical approach to global optimization. Springer Science \& Business Media, 2006

[46] P. A. N. Bosman and D. Thierens, "The balance between proximity and diversity in multiobjective evolutionary algorithms," IEEE Transactions on Evolutionary Computation, vol. 7, no. 2, pp. 174188, 2003.

[47] L. While, P. Hingston, L. Barone, and S. Huband, "A faster algorithm for calculating hypervolume," IEEE Transactions on Evolutionary Computation, vol. 10, no. 1, pp. 29-38, 2006.

[48] H. Ishibuchi, R. Imada, S. Yu, and Y. Nojima, "How to specify a reference point in hypervolume calculation for fair performance comparison," Evolutionary Computation, vol. 26, no. 3, pp. 1-29, 2018.

[49] H. Ishibuchi, R. Imada, Y. Setoguchi, and Y. Nojima, "Reference point specification in inverted generational distance for triangular linear Pareto front," IEEE Transactions on Evolutionary Computation, vol. 22, no. 6, pp. 961-975, 2018.

[50] K. Musselman and J. Talavage, "A tradeoff cut approach to multiple objective optimization," Operations Research, vol. 28, no. 6, pp. 1424-1435, 1980.

[51] E. Besada-Portas, L. d. 1. Torre, J. M. d. 1. Cruz, and A.-T. B. de "Evolutionary trajectory planner for multiple UAVs in realistic scenarios," IEEE Transactions on Evolutionary Computation, vol. 26, no. 4, pp. 619-634, 2010.

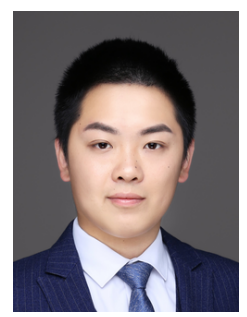

Ye Tian received the B.Sc., M.Sc., and Ph.D. degrees from Anhui University, Hefei, China, in 2012, 2015, and 2018, respectively.

He is currently an Associate Professor with the Institutes of Physical Science and Information Technology, Anhui University, Hefei, China, and also a Postdoctoral Research Fellow with the Department of Computer Science, City University of Hong Kong, Hong Kong. His current research interests include computational intelligence and its applications.

$\mathrm{He}$ is the recipient of the 2018 and 2021 IEEE Transactions on Evolutionary Computation Outstanding Paper Award and the 2020 IEEE Computational Intelligence Magazine Outstanding Paper Award.

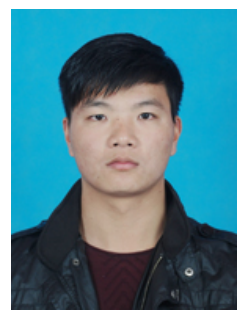

Yajie Zhang received the B.Sc. degree from Anhui University, Hefei, China, in 2018, where he is currently pursuing the Ph.D. degree with the School of Computer Science and Technology, Anhui University, Hefei, China.

His current research interests include constrained evolutionary multiobjective optimization and logistics scheduling.

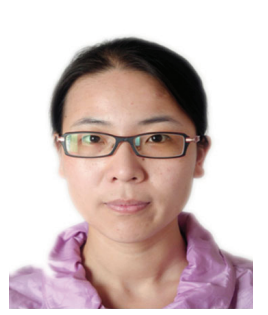

Yansen Su received the B.Sc. degree from Tangshan Normal University, Tangshan, China, in 2007, the M.Sc. degree from Shandong University of Science and Technology, Qingdao, China, in 2010, and the Ph.D. degree from Huazhong University of Science and Technology, Wuhan, China, in 2014.

She is currently an Associate Professor with the School of Computer Science and Technology, Anhui University, Hefei, China. Her main research interests include complexes networks, computational biology, and multi-objective optimization.

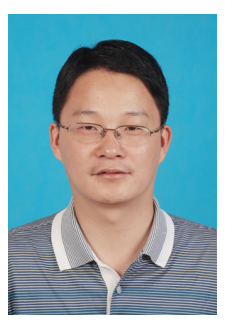

Xingyi Zhang (SM'18) received the B.Sc. degree from Fuyang Normal College, Fuyang, China, in 2003, and the M.Sc. and Ph.D. degrees from Huazhong University of Science and Technology, Wuhan, China, in 2006 and 2009, respectively.

He is currently a Professor with the School of Computer Science and Technology, Anhui University, Hefei, China. His current research interests include unconventional models and algorithms of computation, multi-objective optimization, and membrane computing.

$\mathrm{He}$ is the recipient of the 2018 and 2021 IEEE Transactions on Evolutionary Computation Outstanding Paper Award and the 2020 IEEE Computational Intelligence Magazine Outstanding Paper Award.

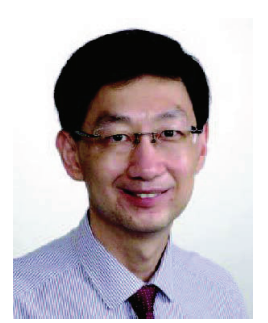

Kay Chen Tan $\left(\mathbf{S M}^{\prime} \mathbf{0 8}-\mathbf{F}^{\prime} \mathbf{1 4}\right)$ received the B.Eng. (First Class Hons.) degree in electronics and electrical engineering and the Ph.D. degree from the University of Glasgow, Glasgow, U.K., in 1994 and 1997, respectively.

He is a Full Professor with the Department of Computer Science, City University of Hong Kong, Hong Kong. He has published over 200 refereed articles and six books.

Prof. Tan is the Editor-in-Chief of the IEEE TRANSACTIONS ON EVOLUTIONARY COMPUTATION, was the Editor-in-Chief of the IEEE Computational Intelligence Magazine from 2010 to 2013, and currently serves as the Editorial Board Member of over 10 journals. He is an elected member of the IEEE CIS AdCom from 2017 to 2019.

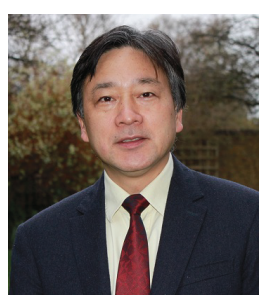

Yaochu Jin (SM'02-F'16) received the B.Sc., M.Sc., and Ph.D. degrees from Zhejiang University, Hangzhou, China, in 1988, 1991, and 1996, respectively, and the Dr.-Ing. degree from Ruhr University Bochum, Bochum, Germany, in 2001.

He is currently a Distinguished Chair Professor in Computational Intelligence, Department of Computer Science, University of Surrey, Guildford, U.K., where he heads the Nature Inspired Computing and Engineering Group. He was a Finland Distinguished Professor and a Changjiang Distinguished Visiting Professor. He has (co)authored over 300 peer-reviewed journal and conference papers and been granted eight patents on evolutionary optimization.

He is the Co-Editor-in-Chief of the IEEE Transactions on Cognitive and Developmental Systems and Complex \& Intelligent Systems. $\mathrm{He}$ is also an Associate Editor or Editorial Board Member of the IEEE Transactions on Evolutionary Computation, IEEE Transactions on Cybernetics, IEEE Transactions on Nanobioscience, Evolutionary Computation, BioSystems, Soft Computing, and Natural Computing. $\mathrm{He}$ is an IEEE Distinguished Lecturer (2017-2019). He is the recipient of the 2014, 2016, and 2020 IEEE Computational Intelligence Magazine Outstanding Paper Award, the 2018 and 2021 IEEE Transactions on Evolutionary Computation Outstanding Paper Award, and the Best Paper Award of the 2010 IEEE Symposium on Computational Intelligence in Bioinformatics and Computational Biology. He has been named a Highly Cited Researcher for 2019 by the Web of Science group. He is a Fellow of IEEE. 